\title{
Analysis of Iterative Methods in Photoacoustic Tomography with Variable Sound Speed
}

\author{
Markus Haltmeier \\ Department of Mathematics, University of Innsbruck \\ Technikestraße 13, A-6020 Innsbruck, Austria \\ E-mail: markus.haltmeier@uibk.ac.at \\ Linh V. Nguyen \\ Department of Mathematics, University of Idaho \\ 875 Perimeter Dr, Moscow, ID 83844, US \\ E-Mail: lnguyen@uidaho.edu
}

\begin{abstract}
In this article, we revisit iterative methods for solving the inverse problem of photoacoustic tomography in free space. Recently, there have been interesting developments on explicit formulations of the adjoint operator, demonstrating that iterative methods is an attractive choice for photoacoustic image reconstruction. In this work, we propose several modifications of current formulations of the adjoint operator which help speed up the convergence and yield improved error estimates. We establish a stability analysis and show that, with our choices of the adjoint operator, the iterative methods can achieve a linear rate of convergence, in the $L^{2}$-norm as well as in the $H^{1}$-norm. In addition, we analyze the normal operator from the microlocal analysis point of view. This gives insight into the convergence speed of the iterative methods and choosing proper weights for the mapping spaces. Finally, we present numerical results using various iterative reconstruction methods for full as well as limited view data. Our results demonstrate that Nesterov's fast gradient and the CG methods converge faster than Landweber's and iterative time reversal methods in the visible as well as the invisible case.
\end{abstract}

Keywords: Photoacoustic tomography, variable sound speed, iterative regularization, adjoint operator, Landweber 's method, Nesterov's method, CG method, visibility condition, invisibility condition, image reconstruction.

AMS Subject Classification: 35R30; 92C55; 65F10; 35A18; 74J05.

\section{Introduction}

Photoacoustic tomography (PAT) is an novel coupled-physics method for non-invasive imaging. It combines the high contrast of optical imaging with the good resolution of ultrasound tomography. The biological object of interest is scanned with a laser light pulse. The photoelastic 
effect induces an acoustic pressure wave propagating in space. One measures the pressure on an observation surface. The aim of PAT is to recover the initial pressure inside the tissue from the measured data. This quantity contains helpful internal information of the object and is the image to be reconstructed.

The mathematical model for PAT is the acoustic wave equation

$$
\left\{\begin{array}{l}
c^{-2}(x) p_{t t}(x, t)-\Delta p(x, t)=0, \quad(x, t) \in \mathbb{R}^{d} \times \mathbb{R}_{+}, \\
p(x, 0)=f(x), \quad p_{t}(x, 0)=0, \quad x \in \mathbb{R}^{d},
\end{array}\right.
$$

where $c: \mathbb{R}^{d} \rightarrow \mathbb{R}$ is the sound speed and $f: \mathbb{R}^{d} \rightarrow \mathbb{R}$ the initial pressure. Let us denote by $S$ the observation surface. We will assume that $S$ is a closed subset of $\partial \Omega$ with nonempty interior $\operatorname{Int}(S)$. Here, $\Omega$ is an open subset of $\mathbb{R}^{d}$ that contains the support of $f$. The mathematical problem of PAT is to invert the map $\mathbf{L}: f \mapsto g:=\left.p\right|_{S \times(0, T)}$. We will refer to this problem as the inverse source problem of PAT.

For our conveniences, let us fix several geometric conventions. We will always assume that the sound speed $c$ is smooth and bounded from below by a positive constant. The space $\mathbb{R}^{d}$ is considered as a Riemannian manifold with the metric $c^{-2}(x) d x^{2}$ and $\Omega$ is assumed to be strictly convex with respect to this metric. Then, all the geodesic rays originating inside $\Omega$ intersect the boundary $\partial \Omega$ at most once. We say that the speed $c$ is nontrapping if all such geodesic rays intersect with $\partial \Omega$; otherwise, the speed $c$ is called trapping. We will denote by $\mathbb{T}^{*} \Omega$ the cotangent bundle of $\Omega$. It can be identified with $\Omega \times \mathbb{R}^{d}$. Also, $\mathbb{T}^{*} \Omega \backslash 0$ is the cotangent bundle of $\Omega$ minus the zero section, which can be identified with $\Omega \times\left(\mathbb{R}^{d} \backslash\{0\}\right)$. A set $V \subset \mathbb{T}^{*} \Omega \backslash 0$ is said to be conic if $(x, \xi) \in V$ implies $(x, \alpha \xi) \in V$ for all $\alpha>0$.

Let us assume that $\operatorname{supp}(f) \subset \Omega_{0}$, where $\Omega_{0} \Subset \Omega$. Then, $\mathbf{L}=\mathbf{L}_{+}+\mathbf{L}_{-}$, where $\mathbf{L}_{ \pm}$are Fourier integral operators (FIOs) of order zero (see [57, Proposition 3]). This fact, in particular, implies that $\mathbf{L}$ is a well-defined linear bounded operator from $H^{s_{1}}\left(\Omega_{0}\right)$ to $H^{s_{2}}(S \times[0, T])$ for all $s_{1} \geq s_{2}$. In this article, we will identify the correct mapping spaces for $\mathbf{L}$ in order to stabilize the inverse problem of PAT and design proper algorithms. For the sake of simplicity, we will assume that $\mathbf{L}$ is injective. Necessary and sufficient conditions for this assumption to hold can be found in [57, Proposition 2].

An essential feature of an inverse problem is its well-posedness or ill-posedness (see [15] and Section 2.1). The inversion of a linear operator $\mathbf{T}$ is called well-posed if the ratio $\|\mathbf{T} x\| /\|x\|$ is bounded from below by a positive constant and ill-posed otherwise. The inverse problem of PAT can be either well-posed or ill-posed, as can be seen in the following two scenarios:

(i) There is a closed subset $S_{0} \subset \partial \Omega$ such that $S_{0} \subset \operatorname{Int}(S)$ and the following condition holds: for any element $(x, \xi) \in \mathbb{T}^{*} \Omega_{0} \backslash 0$, at least one of the unit speed geodesic rays originating from $x$ at time $t=0$ along the direction of $\pm \xi$ intersects with $S_{0}$ at a time $t<T$. This is the so-called visibility condition [41, 67, 32, 50, 57].

(ii) There is an open conic set $V \subset \mathbb{T}^{*} \Omega_{0} \backslash 0$ such that for all $(x, \xi) \in V$ none of the unit speed geodesic rays originating from $x$ at time $t=0$ along the direction of $\pm \xi$ intersects with $S$ at a time $t \leq T$. This is called the invisibility condition.

The visibility and invisibility conditions are almost, but not exactly, complementary. Under the visibility condition, it is shown in [57, Theorem 3] that the inversion of $\mathbf{L}: H_{0}^{1}\left(\Omega_{0}\right) \rightarrow H^{1}(S \times$ 
$[0, T])$ is well-posed. In a similar manner, one can the same result for $\mathbf{L}: L^{2}\left(\Omega_{0}\right) \rightarrow L^{2}(S \times[0, T])$. On the other hand, when the invisibility condition holds, the inversion of $\mathbf{L}: H^{s_{1}}\left(\Omega_{0}\right) \rightarrow H^{s_{2}}(S \times$ $[0, T]$ ) is ill-posed for all $s_{1}, s_{2}$ (see [50]). In this article, we solve the inverse source problem of PAT for both well-posed and ill-posed settings by iterative (regularization) methods. They include Landweber's, Nesterov's and the conjugate gradient (CG) methods. These iterative methods are theoretically convergent to the exact solution in the absence of noise. However, the convergence speed as well as error estimates with respect to the noise level are significantly different for the ill-posed and the well-posed settings.

There exist several methods to solve blem of PAT such as explicit inversion formulas [19, 37, 18, 37, 49, 23, 24, 45, 53], series solutions [38, 2, time reversal [19, 32, 31, 57, and quasi-reversibility [11. Reviews on these methods can be found in [32, 35, 36, 56, 1 To the best of our knowledge, the series solutions, time reversal method, and quasi-reversibility methods only apply to the case when $S$ is a closed surface. Inversion formulas only work for certain closed or flat observation surfaces.

Let us mention that algebraic reconstruction methods have been frequently used for photoacoustic tomography and achieve high quality images (see, e.g., [14, 33, 44, 65, 64, 66]). For example, in [33], the full-wave iterative image reconstruction for photoacoustic tomography with inhomogeneous media was successfully implement and tested with synthetic as well as experimental data. The majority of works on the algebraic methods employ the discretise-then-adjoint approach. In this article we, instead, follow the spirit of [7, 3], where the adjoint operator was explicitly described in the continuous form. Our goal is to systematically analyze the convergence behavior of iterative methods in a continuous framework.

Our approach is most closely related to [7], where Landweber's method was proposed to solve the inverse source problem for PAT. However, we make several changes in order to preserve the well-posedness (when it holds) of the problem and speeds up the convergence speed. For example, instead of considering $\mathbf{L}$ as a mapping from $H_{0}^{1}\left(\Omega_{0}\right)$ to $L^{2}(S \times[0, T])$, we consider $\mathbf{L}$ as a mapping between two Sobolev spaces of the same order (see Section 3). This change not only preserves the well-posedness of the inverse problem under the visibility condition but also makes it simpler to compute the adjoint operator and further speed up the convergence. Additionally, by introducing the weighted norm on the image space, we supply the flexibility to the iterative method. Our choices of mapping spaces are most similar to those in [3], where acoustic measurements on an open set are considered (see also Section 3.2, where we derive theoretical results for this setup). Our adjoint operator is slightly different from that in [3] (although they agree on the $C_{0}^{\infty}$ framework). Moreover, in addition to the $L^{2}$-type product (as considered in [3]), we also consider the adjoint in the $H^{1}$-type product. The analysis for this additional case makes it easier to compare the proposed iterative methods with the well-known Neumann series (i.e., iterative time-reversal) method proposed in [57].

Let us note that our established iterative algorithms converge linearly for the partial data problem under the visibility condition. This convergence rate, to the best of our knowledge, has not been obtained by any previous method. Comparable results, for a different setup of PAT where the acoustic wave is contained in a bounded domain, have recently been obtained in [1, 52, 59.

\footnotetext{
${ }^{1}$ Other setups of PAT that use integrating detectors have been studied in, e.g., [25, 54, 9, 68. However, we do not consider these setups in the present article.
} 
The article is organized as follows. In Section 2, we briefly review several iterative methods that will be used for solving the inverse source problem of PAT. As we will see, the knowledge of the adjoint operator is crucial for those iterative methods. In Section 3 we derive and analyze the adjoint operator $\mathbf{L}^{*}$ of $\mathbf{L}$. We will revisit the PAT with open observation domain in Subsection 3.2. In Section 4, we describe our numerical implementations and present results in various scenarios including the full and partial data cases.

\section{Iterative methods for solving linear equations}

In this section, we briefly review several common iterative methods to solve linear equations in Hilbert spaces; and how to apply them to the inverse source problem of PAT.

\subsection{Iterative methods in Hilbert spaces}

Let $\mathbf{T}: X \rightarrow Y$ be a linear operator mapping between two Hilbert spaces $X$ and $Y$. We denote by $\mathcal{R}(\mathbf{T})$ its range. Assuming that $\mathbf{T}$ is injective, we are interested in inverting $\mathbf{T}$. That is, we want to solve the following problem:

Problem 2.1. Given $y \in \mathcal{R}(\mathbf{T})$, find the solution $x^{*} \in X$ of the equation $\mathbf{T} x=y$.

Problem 2.1 is said to be well-posed if the inverse $\mathbf{T}^{-1}: \mathcal{R}(\mathbf{T}) \rightarrow X$ is bounded and ill-posed otherwise. It can be seen that Problem 2.1 is well-posed if and only if $\mathbf{T}$ is bounded from below, i.e.,

$$
b:=\inf _{x \neq 0} \frac{\|\mathbf{T} x\|_{Y}}{\|x\|_{X}}=\sqrt{\inf _{x \neq 0} \frac{\left\langle\mathbf{T}^{*} \mathbf{T} x, x\right\rangle_{X}}{\|x\|_{X}^{2}}}>0 .
$$

There are several methods to solve Problems 2.1. In this article, we will make use of three methods: Landweber's (see, e.g., [15, Chapter 6]), Nesterov's (see [47, 46]), and the conjugate gradient (CG) methods (see [29, 27, 13, 34, 26, 20, 48, 16, 4, 22, 42]). As we will see, the knowledge of the adjoint operator is essential for all of these iterative methods.

- Landweber's method. The Landweber's method is simply the gradient descent method for minimizing the residual functional $\frac{1}{2}\|\mathbf{T} x-y\|^{2}$. It reads as follows:

$$
x_{k+1}=x_{k}-\gamma \mathbf{T}^{*}\left(\mathbf{T} x_{k}-y\right), \quad k \geq 0,
$$

where $0<\gamma<2 /\|\mathbf{T}\|^{2}$ is a fix relaxation. If the problem is well-posed, the Landweber's method converges linearly. Namely, $\left\|x_{k}-x^{*}\right\|_{X} \leq\left\|\mathbf{I}-\gamma \mathbf{T}^{*} \mathbf{T}\right\|^{k}\left\|x_{0}-x^{*}\right\|_{X}$.

- Nesterov's fast gradient method. Let $L \geq\|\mathbf{T}\|$ and $\mu \leq b$. The Nesterov's algorithm reads as follows (see [46, page 80], and also the original paper [47]) :

1. Initialization: $x_{0}=z_{0}, \alpha_{0} \in[\sqrt{\mu / L}, 1)$, and $q=\frac{\mu}{L}$.

2. While (not stop) do

$$
-x_{k+1}=z_{k}-\frac{1}{L} \mathbf{T}^{*}\left(\mathbf{T} z_{k}-y\right)
$$


- compute $\alpha_{k+1} \in(0,1)$ from the equation $\alpha_{k+1}=\left(1-\alpha_{k+1}\right) \alpha_{k}^{2}+q \alpha_{k+1}$

- set $\beta_{k}=\frac{\alpha_{k}\left(1-\alpha_{k}\right)}{\alpha_{k}^{2}+\alpha_{k+1}}$

$-z_{k+1}=x_{k+1}+\beta_{k}\left(x_{k+1}-x_{k}\right)$

Then [46, Theorem 2.2.3] gives

$$
\left\|\mathbf{T} x_{k}-y\right\|_{Y}^{2} \leq \min \left\{\left(1-\sqrt{\frac{\mu}{L}}\right)^{k}, \frac{4 L}{(2 \sqrt{L}+k \sqrt{\gamma})^{2}}\right\}\left(\left\|\mathbf{T} x_{0}-y\right\|_{Y}^{2}+\gamma\left\|x_{0}-x^{*}\right\|_{X}^{2}\right),
$$

where $\gamma=\frac{\alpha_{0}\left(\alpha_{0} L-\mu\right)}{1-\alpha_{0}}$. If the problem is well-posed and $\mu>0, x_{k}$ converges linearly to $x^{*}$ with the estimate

$$
\left\|x_{k}-x^{*}\right\|_{X} \leq \frac{1}{b}\left(1-\sqrt{\frac{\mu}{L}}\right)^{k}\left(\left\|\mathbf{T} x_{0}-y\right\|_{Y}+\gamma\left\|x_{0}-x^{*}\right\|_{X}\right) .
$$

If $\mu \approx b$, this convergence rate is better than that of Landweber's method (see [46]). When the problem is ill-posed $(b=0)$ or $b$ is unknown, one may choose $\mu=0$ (then the calculation of $\beta_{k}$ can be simplified, e.g., [6]). Although the resulting algorithm is not proven to have a linear convergence rate, it still converges faster than the Landweber's method in our numerical simulations (see Section 4).

- Conjugate gradient (CG) method. We will use the CG method to solve the normal equation $\mathbf{T}^{*} \mathbf{T} x=\mathbf{T}^{*} x$ (see, e.g., [26, Algorithm 2.3]):

1. Initialization: $k=0, r_{0}=y-\mathbf{T} x_{0}, d_{0}=\mathbf{T}^{*} r_{0}$

2. While (not stop) do

$$
\begin{aligned}
& -\alpha_{k}=\left\|\mathbf{T}^{*} r_{k}\right\|_{X}^{2} /\left\|\mathbf{T} d_{k}\right\|_{Y}^{2} \\
& -x_{k+1}=x_{k}+\alpha_{k} d_{k} \\
& -r_{k+1}=r_{k}-\alpha_{k} \mathbf{T} d_{k} \\
& -\beta_{k}=\left\|\mathbf{T}^{*} r_{k+1}\right\|_{X}^{2} /\left\|\mathbf{T}^{*} r_{k}\right\|_{X}^{2} \\
& -d_{k+1}=\mathbf{T}^{*} r_{k+1}+\beta_{k} d_{k}
\end{aligned}
$$

The iterates $x_{k}$ converges to the solution $x^{*}$ of Problem 2.1. When the problem is wellposed, the CG method converges linearly with better convergence rate than Landweber's method. Namely, (see, e.g., [13])

$$
\left\|x_{k}-x\right\|_{X} \leq 2 \frac{\|\mathbf{T}\|}{b}\left(\frac{\|\mathbf{T}\|-b}{\|\mathbf{T}\|+b}\right)^{k}\left\|x_{0}-x\right\|_{X} .
$$

Moreover, assume that $\mathbf{T}^{*} \mathbf{T}=\alpha \mathbf{I}+\mathbf{K}$ where $\mathbf{K}$ is a compact operator. Then, the $\mathrm{CG}$ method is known to converge superlinearly (see, e.g., [28]).

\subsection{Iterative methods for PAT}

In this article, we will study the above three iterative methods for the inverse source problem of PAT. To that end, we need to establish the proper form of the adjoint operator (or equivalently, 
the mapping spaces for $\mathbf{L}$ ), which is done in Section 3. Our goal for the adjoint operator is two-fold: it should be relatively simple to implement and to speed up the convergence. In particular, with our choice of mapping spaces for $\mathbf{L}$, the inverse problem of PAT is well-posed under the visibility condition. Therefore, the linear convergence for the Landweber's and CG method is guaranteed for the exact problem (the same for the Nesterov's method if $\mu>0$ ). This convergence rate, for partial data problem of PAT, has not been obtained before for any other methods. In Section 4, we will implement the iterative methods for PAT. We will also compare them with the iterative time reversal method proposed in [57] (see also [55]).

\section{The adjoint operator for PAT}

Let us recall that $\mathbf{L}: f \mapsto g:=\left.p\right|_{S \times(0, T)}$, where $p$ is defined by the acoustic wave equation (1.1) and $S$ is a closed subset of $\partial \Omega$ with nonempty interior. Our goal is to invert $\mathbf{L}$ using the iterative methods introduced in the previous section. It is crucial to analyze the adjoint operator $\mathbf{L}^{*}$ of L. To that end, we first need to identify the correct mapping spaces for $\mathbf{L}$. We, indeed, will consider two realizations, $\mathbf{L}_{0}$ and $\mathbf{L}_{1}$, of $\mathbf{L}$ corresponding to two different choices of the mapping spaces. To make the presentation rigorous, we will work with several Sobolev spaces and their dual. In particular, we will need to deal with the spaces $L^{2}(O), H^{1}(O), H_{0}^{1}(O)$, and $H^{-1}(O)$ (the dual of $H_{0}^{1}(O)$ ), where $O$ is an open subset in $\mathbb{R}^{d}$. The reader is referred to [40, 17] for the definition and properties of these spaces. As usual, we will identify the space $L^{2}(O)$ with its dual $\left(L^{2}(O)\right)^{\prime}$ (see, e.g., [40, page 31] or [17, page 299]).

We first recall our assumption $\operatorname{supp}(f) \subset \Omega_{0}$, where $\Omega_{0} \Subset \Omega$. Let us denote

$$
\begin{aligned}
& \mathbb{X}_{0}:=\left\{f \in L^{2}\left(\mathbb{R}^{d}\right): \operatorname{supp}(f) \subset \bar{\Omega}_{0}\right\} \\
& \mathbb{X}_{1}:=\left\{f \in H^{1}\left(\mathbb{R}^{d}\right): \operatorname{supp}(f) \subset \bar{\Omega}_{0}\right\} .
\end{aligned}
$$

Then, $\mathbb{X}_{0}$ and $\mathbb{X}_{1}$ are Hilbert spaces with the respective norms

$$
\begin{aligned}
& \|f\|_{\mathbb{X}_{0}}=\left\|c^{-1} f\right\|_{L^{2}\left(\Omega_{0}\right)}, \\
& \|f\|_{\mathbb{X}_{1}}=\|\nabla f\|_{L^{2}\left(\Omega_{0}\right)} .
\end{aligned}
$$

We note that $\mathbb{X}_{0} \simeq L^{2}\left(\Omega_{0}\right)$ and $\mathbb{X}_{1} \simeq H_{0}^{1}\left(\Omega_{0}\right)$ (this second equivalence comes from the Poincaré inequality, see, e.g., [8, Corollary 9.19]). The above chosen norms are convenient for our later purposes.

For the image space, we fix a nonnegative function $\chi \in L^{\infty}(\partial \Omega \times[0, T])$ such that $\operatorname{supp}(\chi)=$ $\Gamma:=S \times[0, T]$ and denote

$$
\begin{aligned}
\mathbb{Y}_{0} & :=\left\{g:\|g\|_{\mathbb{Y}_{0}}:=\|\sqrt{\chi} g\|_{L^{2}(\Gamma)}<\infty\right\} \\
\mathbb{Y}_{1} & :=\left\{g: g(\cdot, 0) \equiv 0,\|g\|_{\mathbb{Y}_{1}}:=\left\|g_{t}\right\|_{\mathbb{Y}_{0}}<\infty\right\} .
\end{aligned}
$$

Let $H^{i}(\Gamma)$ be the standard Sobolev space of order $i$ on $\Gamma$. Noticing that $\mathbf{L}$ is a bounded map from $\mathbb{X}_{i} \rightarrow H^{i}(\Gamma)$ (which follows from [57, Proposition 3]) and $H^{i}(\Gamma) \subset \mathbb{Y}_{i}$, we obtain

Theorem 3.1. For $i=0,1, \mathbf{L}_{i}:=\left.\mathbf{L}\right|_{\mathbb{X}_{i}}$ is a bounded map from $\mathbb{X}_{i}$ to $\mathbb{Y}_{i}$. 
We now consider $\chi g$ as a function on $\partial \Omega \times[0, T]$ which vanishes on $(\partial \Omega \backslash S) \times[0, T]$. The next theorem gives us an explicit formulation of $\mathbf{L}_{i}^{*}$.

Theorem 3.2. Let $g \in C^{\infty}(\Gamma)$.

(a) Consider the wave equation

$$
\left\{\begin{array}{l}
c^{-2}(x) q_{t t}(x, t)-\Delta q(x, t)=0, \quad(x, t) \in\left(\mathbb{R}^{d} \backslash \partial \Omega\right) \times(0, T), \\
q(x, T)=0, \quad q_{t}(x, T)=0, \quad x \in \mathbb{R}^{d}, \\
{[q](y, t)=0,\left[\frac{\partial q}{\partial \nu}\right](y, t)=\chi(y, t) g(y, t), \quad(y, t) \in \partial \Omega \times[0, T] .}
\end{array}\right.
$$

Then

$$
\mathbf{L}_{0}^{*} g=\left.q_{t}(\cdot, 0)\right|_{\Omega_{0}} .
$$

Here and elsewhere, $[\cdot]$ denotes the jump of a function across the boundary $\partial \Omega$. That is

$$
[q]=\left.q_{+}\right|_{\partial \Omega}-\left.q_{-}\right|_{\partial \Omega} \quad \text { and } \quad\left[\frac{\partial q}{\partial \nu}\right]=\left.\frac{\partial q}{\partial \nu}\right|_{\partial \Omega}-\left.\frac{\partial q}{\partial \nu}\right|_{\partial \Omega},
$$

where $q_{+}:=\left.q\right|_{\mathbb{R}^{d} \backslash \bar{\Omega}}$ and $q_{-}:=\left.q\right|_{\Omega}$ have well-defined traces on $\partial \Omega$.

(b) Assume further that $\chi$ is independent of $t$ (i.e., $\chi(y, t)=\chi(y))$. We define

$$
\bar{g}(x, t):=g(x, t)-g(x, T),
$$

and consider the wave equation

$$
\left\{\begin{array}{l}
c^{-2}(x) \bar{q}_{t t}(x, t)-\Delta \bar{q}(x, t)=0, \quad(x, t) \in\left(\mathbb{R}^{d} \backslash \partial \Omega\right) \times(0, T), \\
\bar{q}(x, T)=0, \quad \bar{q}_{t}(x, T)=0, \quad x \in \mathbb{R}^{d}, \\
{[\bar{q}](y, t)=0,\left[\frac{\partial \bar{q}}{\partial \nu}\right](y, t)=\chi(y, t) \bar{g}(y, t), \quad(y, t) \in \partial \Omega \times[0, T] .}
\end{array}\right.
$$

Then,

$$
\mathbf{L}_{1}^{*} g=\Pi\left[\bar{q}_{t}(\cdot, 0)\right],
$$

where $\Pi$ is the projection from $H^{1}\left(\Omega_{0}\right)$ onto $\mathbb{X}_{1}=H_{0}^{1}(\Omega)$.

Let us mention that the projection operator $\Pi$ above is given by $\Pi(f)=f-\phi$. Here, $\phi$ is the harmonic extension of $\left.f\right|_{\partial \Omega_{0}}$ to $\bar{\Omega}_{0}$. That is, $\Delta \phi=0$ in $\Omega_{0}$ and $\left.\phi\right|_{\partial \Omega_{0}}=\left.f\right|_{\partial \Omega_{0}}$. The proof of Theorem 3.2 is similar to that [7, Theorem 1.5]. However, to make our presentation rigorous, we have to employ several results from functional analysis and distribution theory.

Proof. Let us make use of the weak formulation for (3.1) (see Appendix A). Then, for any $v \in C^{\infty}\left(\mathbb{R}^{n} \times \overline{\mathbb{R}}\right)$ such that $v(\cdot, t) \in C_{0}^{\infty}\left(\mathbb{R}^{d}\right)$ for all $t \in \overline{\mathbb{R}}$, we have

$$
\begin{aligned}
\int_{0}^{T}\left(c^{-2} q_{t t}(\cdot, t), v(\cdot, t)\right) d t+\int_{0}^{T} \int_{\mathbb{R}^{d}} \nabla q(x, t) \nabla v(x, t) d x d t= \\
-\int_{0}^{T} \int_{\partial \Omega} \chi(y, t) g(y, t) v(y, t) d y d t .
\end{aligned}
$$


Here, on the first term of the left hand $(\cdot, \cdot)$ is the action of a distribution on a test function. Taking integration by parts with respect to $t$ for the first term and with respect to $x$ for the second term of the left hand side, we obtain

$$
\begin{aligned}
-\left(c^{-2} q_{t}(\cdot, 0), v(\cdot, 0)\right) & +\left(c^{-2} q(\cdot, 0), v_{t}(\cdot, 0)\right) \\
& +\int_{0}^{T} \int_{\mathbb{R}^{d}} q(x, t)\left[c^{-2}(x) v_{t t}(x, t)-\Delta v(x, t)\right] d x d t \\
& =-\int_{0}^{T} \int_{\partial \Omega} \chi(y, t) g(y, t) v(y, t) d y d t .
\end{aligned}
$$

(a) Let $f \in C_{0}^{\infty}\left(\mathbb{R}^{d}\right)$ and $p$ be the solution of 1.1 . Choosing $v=p$ in 3.3 , we obtain

$$
\left(c^{-2} q_{t}(\cdot, 0), f\right)=\int_{0}^{T} \int_{\partial \Omega} \chi(y, t) g(y, t) \mathbf{L}(f)(y, t) d y d t .
$$

For any $f \in C_{0}^{\infty}\left(\Omega_{0}\right)$, the right hand side is bounded by $C\|f\|_{L^{2}\left(\Omega_{0}\right)}$ (since $\mathbf{L}: L^{2}\left(\Omega_{0}\right) \rightarrow$ $L^{2}(\partial \Omega \times[0, T])$ is bounded). Therefore, $c^{-2} q_{t}(\cdot, 0) \in\left(L^{2}\left(\Omega_{0}\right)\right)^{\prime}=L^{2}\left(\Omega_{0}\right)$. Moreover, by the definition of the product in $\mathbb{X}_{0}$ and $\mathbb{Y}_{0}$, we arrive to $\left\langle q_{t}(\cdot, 0), f\right\rangle_{\mathbb{X}_{0}}=\langle g, \mathbf{L}(f)\rangle_{\mathbb{Y}_{0}}$. Since this is true for all $f \in C_{0}^{\infty}\left(\Omega_{0}\right)$, we obtain $\mathbf{L}_{0}^{*} g=\left.q_{t}(\cdot, 0)\right|_{\Omega_{0}}$. This finishes the proof of (a).

(b) Let $\bar{q}$ be the solution of (3.2). Then, $\bar{q}$ satisfies $(3.3)$ with $g$ being replaced by $\bar{g}$. Assuming $f \in C_{0}^{\infty}(\Omega)$ and picking $v=p_{t t}$, we obtain

$$
\left(c^{-2} \bar{q}_{t}(\cdot, 0), p_{t t}(\cdot, 0)\right)=\int_{0}^{T} \int_{\partial \Omega} \chi(y, t) \bar{g}(y, t) \partial_{t}^{2} \mathbf{L}(f)(y, t) d y d t .
$$

Noting that $c^{-2}(x) p_{t t}(x, 0)=\Delta f(x)$, we arrive to

$$
\left(\bar{q}_{t}(\cdot, 0), \Delta f\right)=\int_{0}^{T} \int_{\partial \Omega} \chi(y, t) \bar{g}(y, t) \partial_{t}^{2} \mathbf{L}(f)(y, t) d y d t .
$$

Let us now consider $\chi(y, t)=\chi(y)$. Taking integration by parts for the right hand side with respect to $t$, we get ${ }^{2}$

$$
\left(\bar{q}_{t}(\cdot, 0), \Delta f\right)=-\int_{0}^{T} \int_{\partial \Omega} \chi(y) \bar{g}_{t}(y, t) \partial_{t} \mathbf{L}(f)(y, t) d y d t
$$

That is,

$$
\left(\bar{q}_{t}(\cdot, 0), \Delta f\right)=-\int_{0}^{T} \int_{\partial \Omega} \chi(y) g_{t}(y, t) \partial_{t} \mathbf{L}(f)(y, t) d y d t .
$$

Let us prove that this equation implies $\left.\bar{q}_{t}(\cdot, 0)\right|_{\Omega_{0}} \in H^{1}\left(\Omega_{0}\right)$. To that end, we fix $\Omega^{\prime}$ such that $\Omega_{0} \Subset \Omega^{\prime} \Subset \Omega$. For any $f \in C_{0}^{\infty}\left(\Omega^{\prime}\right)$, the left hand side equals $\left(\Delta \bar{q}_{t}(\cdot, 0), f\right)$ and the right hand side is bounded by $C\|f\|_{H_{0}^{1}\left(\Omega^{\prime}\right)}$ (noting that $\mathbf{L}: H_{0}^{1}\left(\Omega^{\prime}\right) \rightarrow H^{1}(\partial \Omega \times[0, T]$ ) is bounded). We obtain $\Delta \bar{q}_{t}(\cdot, 0) \in H^{-1}\left(\Omega^{\prime}\right)$. Moreover, by the same argument as in $(\mathrm{a}), \bar{q}_{t}(\cdot, 0) \in L^{2}\left(\Omega^{\prime}\right)$. Let $\mu \in C_{0}^{\infty}\left(\Omega^{\prime}\right)$ such that $\mu \equiv 1$ on $\Omega_{0}$ and define $\Psi=\mu \bar{q}_{t}(\cdot, 0)$. Then, $-\Delta \Psi+\Psi \in H^{-1}\left(\Omega^{\prime}\right)$

\footnotetext{
${ }^{2}$ Note that $\bar{g}(\cdot, T) \equiv 0$ and $\partial_{t} \mathbf{L}(f)(\cdot, 0) \equiv 0$.
} 
and $\left.\Psi\right|_{\partial \Omega^{\prime}}=0$. Applying [40, Theorem 9.1 (Chapter 2)], we obtain $\Psi \in H_{0}^{1}\left(\Omega^{\prime}\right)$. Therefore $\left.\bar{q}_{t}(\cdot, 0)\right|_{\Omega_{0}}=\left.\Psi\right|_{\Omega_{0}} \in H^{1}\left(\Omega_{0}\right)$.

Now assume $f \in C_{0}^{\infty}\left(\Omega_{0}\right)$. Taking integration by parts of the left hand side with respect to $x$, we can write 3.4 in the form

$$
\int_{\Omega_{0}} \nabla\left[\bar{q}_{t}(x, 0)\right] \nabla f(x) d x=\int_{0}^{T} \int_{\partial \Omega} \chi(y) g_{t}(y, t) \partial_{t} \mathbf{L}(f)(y, t) d y d t .
$$

Let $\phi$ be the harmonic extension of $\left.\bar{q}_{t}(\cdot, 0)\right|_{\partial \Omega_{0}}$ to $\bar{\Omega}_{0}$. Then, $\bar{q}_{t}(\cdot, 0)-\phi \in H_{0}^{1}\left(\Omega_{0}\right)=\mathbb{X}_{1}$ (see, e.g., [40, Section 2.9]). Moreover,

$$
\begin{aligned}
\int_{\Omega_{0}} \nabla\left[\bar{q}_{t}(x, 0)-\phi(x)\right] \nabla f(x) d x=\int_{\Omega_{0}} \nabla\left[\bar{q}_{t}(x, 0)\right] \nabla f(x) d x+\int_{\Omega_{0}} \Delta \phi(x) f(x) d x \\
=\int_{\Omega_{0}} \nabla\left[\bar{q}_{t}(x, 0)\right] \nabla f(x) d x .
\end{aligned}
$$

We obtain

$$
\int_{\Omega_{0}} \nabla\left[\bar{q}_{t}(x, 0)-\phi(x)\right] \nabla f(x) d x=\int_{0}^{T} \int_{\partial \Omega} \chi(y) g_{t}(y, t) \partial_{t} \mathbf{L}(f)(y, t) d y d t .
$$

That is, $\left\langle\Pi\left[\bar{q}_{t}(\cdot, 0)\right], f\right\rangle_{\mathbb{X}_{1}}=\langle g, \mathbf{L} f\rangle_{\mathbb{Y}_{1}}$, which proves $\mathbf{L}_{1}^{*} g=\Pi\left[\bar{q}_{t}(\cdot, 0)\right]$.

Remark 3.3. Let us make the following observations:

(a) Since $C^{\infty}(\Gamma)$ is dense in both $\mathbb{Y}_{0}$ and $\mathbb{Y}_{1}$, the adjoint operators $\mathbf{L}_{0}^{*}$ and $\mathbf{L}_{1}^{*}$ are uniquely determined by the formulas in Theorem 3.2.

(b) Equation (3.1) can be reformulated as (see A.3):

$$
\left\{\begin{array}{l}
c^{-2}(x) q_{t t}(x, t)-\Delta q(x, t)=-\delta_{\partial \Omega}(x) \chi(x, t) g(x, t), \quad(x, t) \in \mathbb{R}^{d} \times(0, T), \\
q(x, T)=0, \quad q_{t}(x, T)=0, \quad x \in \mathbb{R}^{d} .
\end{array}\right.
$$

This formulation will be used to implement the adjoint operator in Section 4 .

(c) Compared to $\mathbf{L}_{0}^{*}$, the expression for $\mathbf{L}_{1}^{*}$ involves an extra projection operator. In our numerical experiments, we will only use $\mathbf{L}_{0}^{*}$ since it is simpler to implement. However, the knowledge of $\mathbf{L}_{1}^{*}$ is helpful in designing iterative algorithms that converge in the $\mathbb{X}_{1}$ norm (which is equivalent to the $H^{1}$-norm).

(d) If, instead of $\mathbf{L}_{0}: \mathbb{X}_{0} \rightarrow \mathbb{Y}_{0}$, we consider $\mathbf{L}: L^{2}\left(\Omega_{0}\right) \rightarrow \mathbb{Y}_{0}$, then $\mathbf{L}^{*} g=\frac{1}{c^{2}} q_{t}(\cdot, 0)$. Our formulations of $\mathbf{L}^{*}$ are different from [7], where $\mathbf{L}^{*} g=-\Delta^{-1}\left(\frac{1}{c^{2}} q_{t}(\cdot, 0)\right)$. Our formulations make the inverse problem of PAT well-posed under the visibility condition (see Theorem 3.4 below).

Let us recall the visibility condition described in the introduction:

Visibility condition. There is a closed subset $S_{0} \subset \partial \Omega$ such that $S_{0} \subset \operatorname{Int}(S)$ and $T_{0} \leq T$ such that the following condition holds: for any element $(x, \xi) \in \mathbb{T}^{*} \Omega_{0} \backslash 0$, at least one of the unit speed geodesic rays originating from $x$ at time $t=0$ along the directions $\pm \xi$ intersects with $S_{0}$ at a time $t<T_{0}$. 
Let us prove that with our choices of mapping spaces, the inverse problem of PAT is well-posed.

Theorem 3.4. Assume that the visibility condition holds and $\chi>0$ on $S_{0} \times\left[0, T_{0}\right]$. For $i=0,1$, there is a constant $C>0$ such that for any $g=\mathbf{L} f$, we have

$$
\|f\|_{\mathbb{X}_{i}} \leq C\|g\|_{\mathbb{Y}_{i}}
$$

Proof. Let us pick a closed subset $S_{1}$ of $S$ such that $\chi>0$ on $S_{1} \times\left[0, T_{0}\right], S_{0} \subset \operatorname{Int}\left(S_{1}\right)$, and $S_{1} \subset \operatorname{Int}(S)$. Following the lines of [57, Theorem 3], we obtain for $i=0,1^{3}$

$$
\|f\|_{\mathbb{X}_{i}} \leq C\|g\|_{H^{i}\left(S_{1} \times[0, T]\right)} .
$$

Here and elsewhere, $H^{i}\left(S_{1} \times[0, T]\right)$ is the standard Sobolev space of order $i$ on $S_{1} \times[0, T]$ and $C$ is a generic constant which may be different in one place from another.

Let us consider $i=0$. Noticing that $\|g\|_{H^{0}\left(S_{1} \times[0, T]\right)} \leq\|g\|_{\mathbb{Y}_{0}}$, we obtain

$$
\|f\|_{\mathbb{X}_{0}} \leq C\|g\|_{\mathbb{Y}_{0}} .
$$

Let us now consider $i=1$. From $(3.6)$, we have

$$
\|f\|_{\mathbb{X}_{1}} \leq C\|g\|_{H^{1}\left(S_{1} \times[0, T]\right)} .
$$

Let us now prove

$$
\|f\|_{\mathbb{X}_{1}} \leq C\left(\left\|\partial_{t} g\right\|_{L^{2}\left(S_{1} \times[0, T]\right)}+\|g\|_{L^{2}\left(S_{1} \times[0, T]\right)}\right) .
$$

By using a local chart for $\partial \Omega$ if necessary, we can assume without loss of generality that $S_{1}=$ $\mathbb{R}^{d-1}$ and $g=g\left(x^{\prime}, t\right)$ is a compactly supported function on $\mathbb{R}^{d}=\mathbb{R}^{d-1} \times \mathbb{R}$. Let us denote by $\mathcal{H}$ the hyperbolic zone

$$
\mathcal{H}=\left\{\left(x^{\prime}, t, \eta, \tau\right) \in \mathbb{T}^{*}\left(\mathbb{R}^{d-1} \times \mathbb{R}\right): c\left(x^{\prime}\right)|\eta|<\tau\right\} .
$$

Then $\operatorname{WF}(g) \subset \mathcal{H}$ (see, e.g., [57, Proposition 3]). Therefore, the Fourier transform $\hat{g}(\eta, \tau)=$ $\mathcal{F}(g)(\eta, \tau)$ of $g$ decays faster than any powers of $|(\eta, \tau)|$ outside of the region $A:=\{(\eta, \tau):|\tau| \geq$ $\left.c_{0}|\eta|\right\}$, where $c_{0}=\min _{x} c(x)$. We denote by $\chi_{A}$ the characteristic function of $A$ and define

$$
\mathbf{T}(f)=\mathcal{F}^{-1}\left(\hat{g}(\eta, \tau) \chi_{A}(\eta, \tau)\right), \quad \mathbf{K}(f)=\mathcal{F}^{-1}\left(\hat{g}(\eta, \tau)\left(1-\chi_{A}(\eta, \tau)\right) .\right.
$$

Then $\mathbf{T}$ and $\mathbf{K}$ are bounded operators from $\mathbb{X}_{1}$ to $H^{1}\left(\mathbb{R}^{d}\right)$ and $\mathbf{T}+\mathbf{K}=\mathbf{L}$. Since $\mathcal{F}(\mathbf{K} f)(\eta, \tau)=$ $\hat{g}(\eta, \tau)\left(1-\chi_{A}(\eta, \tau)\right)$ decays faster than any powers of $|(\eta, \tau)|$, we obtain $\mathcal{R}(\mathbf{K}) \subset H^{s}\left(\mathbb{R}^{d}\right)$ for any $s>0$. Therefore, $\mathbf{K}$ is a compact operator. Moreover, from (3.7), we obtain

$$
\|f\|_{\mathbb{X}_{1}} \leq C\left(\|\mathbf{T} f\|_{H^{1}\left(\mathbb{R}^{d}\right)}+\|\mathbf{K} f\|_{H^{1}\left(\mathbb{R}^{d}\right)}\right) \leq C\left(\|(\mathbf{T} f, g)\|_{H^{1}\left(\mathbb{R}^{d}\right) \times L^{2}\left(\mathbb{R}^{d}\right)}+\|\mathbf{K} f\|_{H^{1}\left(\mathbb{R}^{d}\right)}\right) .
$$

Since $f \rightarrow(\mathbf{T} f, g)$ is injective, applying [61, Theorem V.3.1], we obtain

$$
\|f\|_{\mathbb{X}_{1}} \leq C\|(\mathbf{T} f, g)\|_{H^{1}\left(\mathbb{R}^{d}\right) \times L^{2}\left(\mathbb{R}^{d}\right)} .
$$

\footnotetext{
${ }^{3}$ The result for $i=1$ is obtained in that reference. The result for $i=0$ is obtained similarly, one only needs to invoke [39, Theorem 2.3] instead of [39, Theorem 2.1].
} 
We note that

$$
\begin{aligned}
\|\mathbf{T} f\|_{H^{1}\left(\mathbb{R}^{d}\right)}^{2}=\int_{A}|\hat{g}(\eta, \tau)|^{2}\left(1+|\eta|^{2}+|\tau|^{2}\right) d \eta d \tau \leq C \int_{\mathbb{R}^{d}}|\hat{g}(\eta, \tau)|^{2}\left(1+|\tau|^{2}\right) d \eta d \tau & =C\left(\|g\|_{L^{2}\left(\mathbb{R}^{d}\right)}^{2}+\left\|g_{t}\right\|_{L^{2}\left(\mathbb{R}^{d}\right)}^{2}\right) .
\end{aligned}
$$

Therefore,

$$
\|f\|_{\mathbb{X}_{1}} \leq C\left(\left\|\partial_{t} g\right\|_{L^{2}\left(\mathbb{R}^{d}\right)}+\|g\|_{L^{2}\left(\mathbb{R}^{d}\right)}\right) .
$$

This finishes the proof of 3.8 ).

Keeping in mind that $g(\cdot, 0) \equiv 0$, we obtain from $(3.8)$

$$
\|f\|_{\mathbb{X}_{1}} \leq C\left\|\partial_{t} g\right\|_{L^{2}\left(S_{1} \times[0, T]\right)} .
$$

That is,

$$
\|f\|_{\mathbb{X}_{1}} \leq C\|g\|_{\mathbb{Y}_{1}},
$$

which concludes our proof.

Remark 3.5. Let us recall that (see Section 2.1) when the linear inverse problem is well-posed, Landweber's and the CG methods have a linear rate of convergence. Theorem 3.4 shows that with our choices of mapping spaces, the inverse problem of PAT is well-posed under the visibility condition. Therefore, Landweber's and the CG methods converge linearly in either $L^{2}$-norm (i.e., $\mathbb{X}_{0}$-norm) or $H^{1}$-norm (i.e., $\mathbb{X}_{1}$-norm), depending on our choice of the adjoint operator in Theorem 3.2, if the visibility condition holds 4 . This convergence rate has not been obtained before by any method.

\subsection{Microlocal analysis for the normal operator $\mathbf{L}^{*} \mathbf{L}$}

To better understand the nature of $\mathbf{L}^{*} \mathbf{L}$, we will analyze it from the microlocal analysis point of view. Let us recall that $r_{ \pm}(x, \xi)$ is the (unit speed) geodesic rays originating from $x$ along direction of $\pm \xi$. We assume that $r_{ \pm}(x, \xi)$ intersects the boundary $\partial \Omega$ at a unique point $x_{ \pm}=$ $x_{ \pm}(x, \xi)$. We denote by $\theta_{ \pm}$the angle between $r_{ \pm}(x, \xi)$ and the normal vector of $\partial \Omega$ at $x_{ \pm}$. Our main result is the following theorem.

Theorem 3.6. Assume that $\chi \in C_{0}^{\infty}(\partial \Omega \times[0, T])$ and $\mathbf{L}=\mathbf{L}_{0}$. Then, the normal operator $\mathbf{N}=\mathbf{L}^{*} \mathbf{L}$ is a pseudo-differential operator of order zero, whose principal symbol is

$$
\sigma_{0}(x, \xi)=\frac{1}{4}\left(\frac{c\left(x_{+}\right) \chi\left(x_{+}, t_{+}\right)}{\cos \left(\theta_{+}\right)}+\frac{c\left(x_{-}\right) \chi\left(x_{-}, t_{-}\right)}{\cos \left(\theta_{-}\right)}\right) .
$$

Here, $t_{ \pm}$is the geodesics distance between $x$ and $x_{ \pm}$.

Let us note that it may happen that one (or both) of the geodesic rays $r_{ \pm}(x, \xi)$ does not intersect $\partial \Omega$ (that is, speed is trapping). In that case, Theorem 3.6 still holds if we replace $\chi\left(x_{ \pm}, t_{ \pm}\right)$by 0 .

\footnotetext{
${ }^{4}$ The same conclusion holds for the Nesterov's method if $\mu>0$.
} 
Proof. We first intuitively describe the effect of $\mathbf{L}^{*} \mathbf{L}$ on the wave front set of a function $f$ supported inside $\bar{\Omega}_{0}$. For simplicity, we assume that $f$ is microlocally supported near an element $(x, \xi) \in \mathbb{T}^{*} \Omega \backslash 0$. Let us analyze the effect of $\mathbf{L}$ to $f$ by considering the wave equation (1.1). At time $t=0$, the singularity of $f$ at $(x, \xi)$ breaks into two equal parts (see [57, page 7]). They induce the singularities of $p$ on the bicharacteristic rays $\mathcal{C}_{ \pm}(x, \xi)$ originating at $(x, 0, \xi, \tau=$ $c(x)|\xi|)$ and $(x, 0,-\xi, \tau=c(x)|\xi|)$ (see [30]). The projection of each bicharacteristic ray $\mathcal{C}_{ \pm}(x, \xi)$ on the spatial domain $\mathbb{R}^{d}$ is the geodesic ray $r_{ \pm}(x, \xi)$ on $\mathbb{R}^{d}$ (recalling that $\mathbb{R}^{d}$ is equipped with the metric $\left.c^{-2}(x) d x^{2}\right)$. Each of the geodesic ray hits the boundary $\partial \Omega$ at a unique point $x_{ \pm}$ and time $t_{ \pm}$. The corresponding singularity of $p$ at $\left(x_{ \pm}, t_{ \pm}\right)$is denoted by $\left(x_{ \pm}, t_{ \pm}, \xi_{ \pm}, \tau_{ \pm}\right)$. Its projection on $\mathbb{T}_{\left(x_{ \pm}, t_{ \pm}\right)}^{*}(\partial \Omega \times[0, T])$ induces a singularity of $g$ at $\left(x_{ \pm}, t_{ \pm}, \eta_{ \pm}, \tau_{ \pm}\right)$. Now, consider the adjoint equation (3.1) which defines $\mathbf{L}^{*}$. The singularity of $g$ at $\left(x_{ \pm}, t_{ \pm}, \eta_{ \pm}, \tau_{ \pm}\right)$then induces two singularities of $q$ at $\left(x_{ \pm}, t_{ \pm}, \xi^{\prime}, \tau_{ \pm}\right)$. Here, $\xi^{\prime}=\eta_{ \pm} \pm \sqrt{c^{-2}\left(x_{ \pm}\right) \tau^{2}-\left|\eta_{ \pm}\right|^{2}} \nu$ where $\nu$ is the normal vector of $\partial \Omega$ at $x_{ \pm}$(note that one of the such $\xi^{\prime}$ equals $\xi_{ \pm}$). These two singularities propagate along two opposite directions when going backward in time, one into the domain $\Omega$ (along the ray $\mathcal{C}_{ \pm}(x, \xi)$ but in the negative direction) and one away from $\Omega$. At $t=0$ the first one lands back to $(x, \xi)$ and the other one lands outside of $\Omega$. This shows the pseudo-locality of $\mathbf{L}^{*} \mathbf{L}:\left.f \rightarrow q_{t}(\cdot, 0)\right|_{\Omega_{0}}$ and heuristically explains that $\mathbf{L}^{*} \mathbf{L}$ is a pseudo-differential operator. Our rigorous argument follows below.

Let us recall that up to a smooth term (e.g., 63]):

$$
p(x, t)=\frac{1}{(2 \pi)^{d}} \sum_{\sigma= \pm} \int e^{i \phi_{\sigma}(x, t, \xi)} a_{\sigma}(x, t, \xi) \hat{f}(\xi) d \xi=: p_{+}(x, t)+p_{-}(x, t) .
$$

The phase function $\phi_{\sigma}, \sigma= \pm$, satisfies the eikonal equation

$$
\partial_{t} \phi_{\sigma}(x, t, \xi)+\sigma\left|\nabla_{x} \phi_{\sigma}(x, t, \xi)\right|=0, \quad \phi_{\sigma}(x, 0, \xi)=x \cdot \xi .
$$

The amplitude function $a_{\sigma}$ satisfies

$$
a(x, t, \xi) \sim \sum_{m=0}^{\infty} a_{-m}(x, t, \xi)
$$

where $a_{-m}$ is homogeneous of order $-m$ in $\xi$. The leading term $a_{0}=a(x, t, \xi)$ satisfies the transport equation ${ }^{5}$

$$
\left(\partial_{t} \phi_{\sigma} \partial_{t}-c^{2}(x) \nabla_{x} \phi_{\sigma} \cdot \nabla_{x}\right) a_{0}(x, t, \xi)=0,
$$

with the initial condition $a_{0}(x, \xi, 0)=1 / 2$.

Then, up to a smooth term, we obtain $g=\left.\left(p_{+}+p_{-}\right)\right|_{\partial \Omega}=: g_{+}+g_{-}$. Solving the adjoint problem (3.1), we obtain, up to a smooth term, $\mathbf{L}^{*} \mathbf{L} f=\partial_{t} q_{+}(\cdot, 0)+\partial_{t} q_{-}(\cdot, 0)$. Here, $q_{\sigma}$ (for $\sigma= \pm$ ) is defined by

$$
\left\{\begin{array}{l}
c^{-2}(x) q_{\sigma, t t}(x, t)-\Delta q_{\sigma}(x, t)=0, \quad(x, t) \in\left(\mathbb{R}^{d} \backslash \partial \Omega\right) \times(0, T), \\
q_{\sigma}(x, T)=0, \quad q_{\sigma, t}(x, T)=0, \quad x \in \mathbb{R}^{d}, \\
{\left[q_{\sigma}\right](y, t)=0,\left[\frac{\partial q_{\sigma}}{\partial \nu}\right](y, t)=\chi(y, t) g_{\sigma}(y, t), \quad(y, t) \in \partial \Omega \times(0, T) .}
\end{array}\right.
$$

\footnotetext{
${ }^{5}$ In several references, the equation contains a zero order term. However, that term turns out to be zero.
} 
Let us show that $f \rightarrow f_{+}:=\partial_{t} q_{+}(\cdot, 0)$ is a pseudo-differential operator with the principal symbol

$$
\sigma_{+}(x, \xi)=\frac{1}{4} \frac{\chi\left(x_{+}, t_{+}\right)}{\cos \left(\theta_{+}\right)} .
$$

We recall that $x_{+}$is the intersection of the positive geodesic ray $r_{+}(x, \xi)$ and $\partial \Omega$, and $t_{+}$is the time to travel along the geodesic from $x$ to $x_{+}$. Let $\left(x_{+}, t_{+}, \xi_{+}, \tau_{+}\right)$be the corresponding element on the bicharacteristic and $\left(x_{+}, t_{+}, \eta_{+}, \tau_{+}\right)$its projection on $\mathbb{T}_{\left(x_{+}, t_{+}\right)}^{*}(\partial \Omega \times[0, T])$. Then, $\left(x_{+}, t_{+}, \eta_{+}, \tau_{+}\right)$is in the hyperbolic zone, that is $\tau_{+}>c\left(x_{+}\right)\left|\eta_{+}\right|$. Let us show that the mapping $\left.\left.q_{+}\right|_{\partial \Omega \times[0, T]} \rightarrow\left[\partial_{\nu} q_{+}\right]\right|_{\partial \Omega \times[0, T]}$ is an elliptic pseudo-differential operator near $\left(x_{+}, t_{+}, \eta_{+}, \tau_{+}\right)$.

Indeed, for simplicity, we assume that locally near $x_{+}, \partial \Omega$ is flat, and $h=\left.q_{+}\right|_{\partial \Omega \times[0, T]}$ is supported near $\left(x_{+}, t_{+}\right)$. We then can write $y=\left(y^{\prime}, 0\right)$ for all $y \in \partial \Omega$ and assume that $\Omega \subset\left\{x \in \mathbb{R}^{d}: x_{n}<\right.$ $0\}$. The parametrix $q_{\text {in }}$ (respectively $q_{\text {out }}$ ) for $q_{+}$in $\Omega$ (respectively $\Omega^{c}$ ) near $\left(x_{+}, t_{+}\right)$is of the form

$$
q_{\text {in } / \text { out }}(x, t)=\frac{1}{(2 \pi)^{d}} \sum_{s=F, B} \int_{\mathbb{R}} \int_{\mathbb{R}^{d-1}} e^{i \psi_{s}(x, t, \eta, \tau)} d_{s}(x, t, \eta, \tau) \hat{h}(\eta, \tau) d \eta d \tau .
$$

Here,

$$
d_{F}(y, t, \eta, \tau)+d_{B}(y, t, \eta, \tau)=1, \quad \psi_{s}(y, t, \eta, \tau)=y^{\prime} \cdot \eta-t \tau, \quad y \in \partial \Omega
$$

and

$$
\hat{h}(\eta, \tau)=\int_{\mathbb{R}} \int_{\mathbb{R}^{d-1}} h\left(y^{\prime}, 0, t\right) e^{i(-\eta \cdot y+t \tau)} d y^{\prime} d t .
$$

Similarly to $\phi_{+}$, the phase function $\psi=\psi_{F, B}$ satisfies the eikonal equation

$$
\left|\partial_{t} \psi(x, t, \eta, \tau)\right|=c(x)\left|\nabla_{x} \psi(x, t, \eta, \tau)\right| .
$$

In particular, we obtain

$$
\partial_{x_{n}} \psi_{F}(y, t, \eta, \tau)=\sqrt{c^{-2}(y) \tau^{2}-\eta^{2}}, \quad \partial_{x_{n}} \psi_{B}(y, t, \eta, \tau)=-\sqrt{c^{-2}(y) \tau^{2}-\eta^{2}}, \quad y \in \partial \Omega .
$$

Roughly speaking, the phase function $\psi_{F}$ transmits (forward) wave from left to right (along the $x_{n}$ direction) and $\psi_{B}$ transmits (backward) wave to the opposite direction. Since $q(x, T)=$ $q_{t}(x, T)=0$ for all $x \in \mathbb{R}^{d}$, we obtain that there is no backward wave inside $\Omega$ and no forward wave outside $\Omega$. That is,

$$
q_{\text {in }}(x, t)=\frac{1}{(2 \pi)^{d}} \int_{\mathbb{R}} \int_{\mathbb{R}^{d-1}} e^{i \psi_{F}(x, t, \xi)} d_{\mathrm{in}}(x, t, \eta, \tau) \hat{h}(\eta, \tau) d \eta d \tau,
$$

and

$$
q_{\text {out }}(x, t)=\frac{1}{(2 \pi)^{d}} \int_{\mathbb{R}} \int_{\mathbb{R}^{d-1}} e^{i \psi_{B}(x, t, \xi)} d_{\text {out }}(x, t, \eta, \tau) \hat{h}(\eta, \tau) d \eta d \tau \text {. }
$$

Moreover,

$$
d_{\text {in }}(y, t, \eta, \tau)=d_{\text {out }}(y, t, \eta, \tau)=1, \quad y \in \partial \Omega .
$$

Up to lower order terms, we obtain micirolocally near the hyperbolic element $\left(x_{+}, t_{+}, \eta_{+}, \tau_{+}\right)$

$$
\left[\partial_{x_{n}} q_{+}\right](y, t)=\frac{1}{(2 \pi)^{d}} \int_{\mathbb{R}} \int_{\mathbb{R}^{d-1}} e^{i\left(y^{\prime} \cdot \eta-t \tau\right)}(-2 i) \sqrt{c^{-2}(y) \tau^{2}-\eta^{2}} \hat{h}(\eta, \tau) d \eta d \tau .
$$


That is, the mapping $\left.q_{+}\right|_{\partial \Omega} \rightarrow\left[\partial_{\nu} q_{+}\right]$is an elliptic pseudo-differential operator at the element $\left(x_{+}, t_{+}, \eta_{+}, \tau_{+}\right)$with principal symbol $(-2 i) \sqrt{c^{-2}\left(x_{+}\right) \tau_{+}^{2}-\eta_{+}^{2}}$. Therefore, the mapping $\left.\left[\partial_{\nu} q_{+}\right]_{\partial \Omega \times[0, T]} \rightarrow q_{+}\right|_{\partial \Omega \times[0, T]}$ is also a pseudo-differential operator near $\left(x_{+}, t_{+}, \eta_{+}, \tau_{+}\right)$with the principal symbol $\frac{1}{(-2 i) \sqrt{c^{-2}\left(x_{+}\right) \tau_{+}^{2}-\left|\eta_{+}\right|^{2}}}$.

Noting that $f \rightarrow \chi g_{+}$and $\left.\left.q_{+}\right|_{\partial \Omega \times[0, T]} \rightarrow q_{+}(\cdot, t)\right|_{\Omega}$ are FIOs, we obtain $\left.f \rightarrow q_{+}(\cdot, t)\right|_{\Omega}$ is also an FIO. We, hence, can write the parametrix for $q_{+}$in $\Omega$ in the form

$$
q_{+}(x, t)=\frac{1}{(2 \pi)^{d}} \int_{\mathbb{R}^{d}} e^{i \phi_{+}(x, t, \xi)} b(x, t, \xi) \hat{f}(\xi) d \xi
$$

In particular,

$$
q_{+}(y, t)=\frac{1}{(2 \pi)^{d}} \int_{\mathbb{R}^{d}} e^{i \phi_{+}(y, t, \xi)} b(y, t, \xi) \hat{f}(\xi) d \xi, \quad y \in \partial \Omega .
$$

On the other hand,

$$
\chi(y, t) g_{+}(y, t)=\frac{1}{(2 \pi)^{d}} \int_{\mathbb{R}^{d}} e^{i \phi_{+}(y, t, \xi)} \chi(y, t) a(y, t, \xi) \hat{f}(\xi) d \xi, \quad y \in \partial \Omega .
$$

Since $\chi g_{+}=\left[\partial_{\nu} q_{+}\right] \rightarrow q_{+}$is a pseudo-differential with principal symbol $\frac{1}{(-2 i) \sqrt{c^{-2}\left(x_{+}\right) \tau_{+}^{2}-\left|\eta_{+}\right|^{2}}}$ at $\left(x_{+}, t_{+}, \eta_{+}, \tau_{+}\right)$, the principal part $b_{p}$ of $b$ satisfies

$$
b_{p}\left(x_{+}, t_{+}, \xi_{+}\right)=-\frac{\chi\left(x_{+}, t_{+}\right)}{2 i \sqrt{c^{-2}\left(x_{+}\right) \tau_{+}^{2}-\left|\eta_{+}\right|^{2}}} a_{0}\left(x_{+}, \xi_{+}, t_{+}\right) .
$$

Since $b_{\text {in }}$ and $a_{0}$ satisfy the same transport equation on the geodesic ray $r_{+}(x, \xi)$, the above equation implies

$$
b_{p}(x, 0, \xi)=-\frac{\chi\left(x_{+}, t_{+}\right)}{2 i \sqrt{c^{-2} \tau_{+}^{2}-\left|\eta_{+}\right|^{2}}} a_{0}(x, 0, \xi)=-\frac{\chi\left(x_{+}, t_{+}\right)}{4 i \sqrt{c^{-2} \tau_{+}^{2}-\left|\xi_{+}\right|^{2}}} .
$$

Noting that $\partial_{t} \phi_{+}(x, 0, \xi)=-c(x)\left|\nabla_{x} \phi_{+}(x, 0, \xi)\right|=-c(x)|\xi|$, we obtain, from (3.12), up to lower order terms,

$$
f_{+}(x)=\frac{1}{(2 \pi)^{d}} \int_{\mathbb{R}^{d}} e^{i x \cdot \xi} \frac{c(x)|\xi| \chi\left(x_{+}, t_{+}\right)}{4 \sqrt{c^{-2}\left(x_{+}\right) \tau_{+}^{2}-\left|\xi_{+}^{\prime}\right|^{2}}} \hat{f}(\xi) d \xi .
$$

Noting that $c(x)|\xi|=\left.c\left(x_{+}\right)\left|\xi_{+}\right|\right|^{6}$ we obtain the mapping $f \rightarrow f_{+}$is a pseudo-differential operator with principal symbol

$$
\frac{c(x)|\xi| \chi\left(x_{+}, t_{+}\right)}{4 \sqrt{c^{-2}\left(x_{+}\right) \tau_{+}^{2}-\left|\eta_{+}\right|}}=\frac{c\left(x_{+}\right)\left|\xi_{+}\right| \chi\left(x_{+}, t_{+}\right)}{4 \sqrt{\left|\xi_{+}\right|^{2}-\left|\eta_{+}\right|}}=\frac{c\left(x_{+}\right) \chi\left(x_{+}, t_{+}\right)}{4 \cos \left(\theta_{+}\right)} .
$$

Repeating the above argument for $f \rightarrow f_{-}$, we finish the proof.

Remark 3.7. Let us make the following observations:

\footnotetext{
${ }^{6}$ This comes from the fact that $c(x)|\xi|=\tau$ and $\tau$ is constant on the bicharacteristic rays (see, e.g., [50]).
} 
(a) The calculus of symbols can be explained more intuitively by considering the current set up as the limit of the open set measurement. This will be discussed in Section 3.2.

(b) We notice that the function $\chi$ plays the role of preconditioning for the inverse problem of PAT. Formula (3.9) may give us some hint on how to make a good choice of $\chi$. Indeed, let us consider the case $c=1, S$ is the sphere of radius $R$, and $\chi(y, t)=t$. Since the geodesics are straight lines, we observe that $\theta_{+}=\theta_{-}=\theta$ and hence

$$
\sigma_{0}(x, \xi)=\frac{t_{+}+t_{-}}{4 \cos \theta}
$$

We notice that $t_{+}+t_{-}$is the length of the line segment connecting $x_{+}$and $x_{-} . A$ simple geometric observation then gives:

$$
\sigma_{0}(x, \xi)=\frac{R}{2} .
$$

We obtain

$$
\mathbf{L}^{*} \mathbf{L}=\frac{R}{2} \mathbf{I}+\mathbf{K}
$$

where $\mathbf{K}$ is a compact operator. Therefore, in such a situation, the CG method for the inverse problem of PAT converges superlinearly (see the discussion of the CG method in Section 2.1). We note that (3.13) can be derived from the results in [19, 18]. Indeed, for odd $d$, [19] even gives $\mathbf{L}^{*} \mathbf{L}=\frac{R}{2} \mathbf{I}$.

The above discussion also suggests the choice of $\chi(y, t)=t$ when the speed is almost constant. The in-depth discussion on the preconditioning, however, is beyond the scope of this article.

Let us recall the time-reversal technique for PAT (see, e.g., [19, 32, 57]). Consider the time reversal wave equation

$$
\left\{\begin{array}{l}
c^{-2}(x) q_{t t}(x, t)-\Delta q(x, t)=0, \quad(x, t) \in \Omega \times[0, T], \\
q(x, T)=\phi(x), \quad q_{t}(x, T)=0, \quad x \in \Omega, \\
q(x, t)=\chi(x, t) g(x, t), \quad(x, t) \in \partial \Omega \times[0, T] .
\end{array}\right.
$$

Here, $\phi$ is the harmonic extension of $\left.\chi(x, T) g(x, T)\right|_{x \in \partial \Omega}$ to $\bar{\Omega}$. The time-reversal operator is defined by $\Lambda g=q(\cdot, 0)$. It is proved in [57, Theorem 1] that, if $\chi \equiv 1$,

$$
\|\mathbf{I}-\Lambda \mathbf{L}\|<1 .
$$

This suggest that $\Lambda \mathbf{L}$ can be used as the first step for a iterative method (see [57, Theorem 1]); it is called iterative time reversal method or Neumann series solution (see also [55] for the thorough numerical discussion and [58] for nonsmooth sound speed). It is shown in [57] that $\Lambda \mathbf{L}$ is a pseudodifferential operator of order zero with the principal symbol

$$
\sigma_{0}(x, \xi)=\frac{1}{2}\left(\chi\left(x_{+}, t_{+}\right)+\chi\left(x_{+}, t_{-}\right)\right) .
$$

This is different from the symbol of $\mathbf{L}^{*} \mathbf{L}$ shown in Theorem 3.6. We, in particular, conclude that the adjoint operator $\mathbf{L}^{*}$ is fundamentally different from the time reversal operator $\Lambda$. We also note that no proof for the convergence of iterative time reversal method is available for limited data problem, even under the visibility condition. However, numerically it works reasonably well in this situation (as demonstrated in [55] and also Section 4). 


\subsection{Open domain observations revisited}

Let us consider the setup used in [3]. Namely, let us consider the operator $\mathbf{L}_{\omega}$ defined by

$$
\left(\mathbf{L}_{\omega} f\right)(x, t)=\omega(x, t) p(x, t) .
$$

Here, $p$ is the solution of 1.1 with initial pressure $f$, and $0 \leq \omega \in C^{\infty}\left(\mathbb{R}^{d} \times[0, T]\right)$ is the window function, whose support determines the accessible region for the data. We assume that $\operatorname{supp}(\omega)=\mathrm{B} \times[0, T]$, where $\mathrm{B} \subset \mathbb{R}^{d}$ is an open band. That is, $\mathbb{R}^{d} \backslash \overline{\mathrm{B}}=\Omega \cup \Omega^{\prime}$, where $\Omega \cap \Omega^{\prime}=\emptyset$, $\Omega$ is bounded and $\Omega^{\prime}$ is unbounded. We again, assume that $f$ is supported in $\bar{\Omega}_{0}$ where $\Omega_{0} \Subset \Omega$, and are interested in the problem of finding $f$ given $\mathbf{L}_{\omega} f$. It can be solved by the iterative methods described in Section 2.1, which we do not elaborate further in this article. We, instead, focus on analyzing the adjoint operator in this setup.

We propose the following method to compute the adjoint of $\mathbf{L}_{\omega}$. Consider the time-reversed problem

$$
\left\{\begin{array}{l}
c^{-2}(x) q_{t t}(x, t)-\Delta q(x, t)=-\omega(x, t) h(x, t), \quad(x, t) \in \mathbb{R}^{d} \times(0, T), \\
q(x, T)=0, \quad q_{t}(x, T)=0, \quad x \in \mathbb{R}^{d}
\end{array}\right.
$$

We define

$$
\mathbf{L}_{\omega}^{*}(h)=\partial_{t} q(\cdot, 0) \text {. }
$$

Let $\bar{h}(\cdot, t)=h(\cdot, t)-h(\cdot, T)$ and $\bar{q}$ be the solution of $(3.14)$ with $h$ being replaced by $\bar{h}$. We define (recalling that $\Pi$ denotes the projection from $\mathbb{X}_{1}$ onto $H_{0}^{1}\left(\Omega_{0}\right)$ )

$$
\overline{\mathbf{L}}_{\omega}^{*}(h)=\Pi\left[\bar{q}_{t}(\cdot, 0)\right] .
$$

Let us recall the space $\mathbb{X}_{i}$ defined at the beginning of Section 3 . Similarly to the spaces $\mathbb{Y}_{i}$, we define

$$
\begin{array}{ll}
\widetilde{\mathbb{Y}}_{0} & :=\left\{h:\|h\|_{\widetilde{\mathbb{Y}}_{0}}:=\|\sqrt{\omega} h\|_{L^{2}(\mathrm{~B} \times[0, T])}<\infty\right\}, \\
\widetilde{\mathbb{Y}}_{1} & :=\left\{h: h(\cdot, 0) \equiv 0 \text { in B },\|h\|_{\widetilde{\mathbb{Y}}_{1}}:=\left\|h_{t}\right\|_{\widetilde{\mathbb{Y}}_{0}}<\infty\right\} .
\end{array}
$$

The following lemma shows that $\mathbf{L}_{\omega}^{*}$ and $\overline{\mathbf{L}}_{\omega}^{*}$ are the adjoints of $\mathbf{L}_{\omega}$, given the correct mapping spaces.

Theorem 3.8. We have

(a) For all $f \in H_{0}(\Omega)$ and $h \in \widetilde{\mathbb{Y}}_{0}$,

$$
\left\langle\mathbf{L}_{\omega} f, h\right\rangle_{\widetilde{\mathbb{Y}}_{0}}=\left\langle f, \mathbf{L}_{\omega}^{*} h\right\rangle_{\mathbb{X}_{0}} .
$$

That is, $\mathbf{L}_{\omega}^{*}$ is the adjoint of $\mathbf{L}_{\omega}: \mathbb{X}_{0} \rightarrow \widetilde{\mathbb{Y}}_{0}$.

(b) Assume that $\omega$ is independent of $t$, then for all $f \in H_{1}(\Omega)$ and $h \in \widetilde{\mathbb{Y}}_{1}$,

$$
\langle\mathbf{L} f, h\rangle_{\widetilde{\mathbb{Y}}_{1}}=\left\langle f, \overline{\mathbf{L}}_{\omega}^{*} h\right\rangle_{\mathbb{X}_{1}} .
$$

That is, $\overline{\mathbf{L}}_{\omega}^{*}$ is the adjoint of $\mathbf{L}_{\omega}: \mathbb{X}_{1} \rightarrow \widetilde{\mathbb{Y}}_{1}$. 
The proof of Theorem 3.8 is similar to that of Theorem 3.2. We skip it for the sake of brevity. Let us notice that our definition of $\mathbf{L}_{\omega}^{*}$ is slightly different from [3]. It is motivated by case of the observation on a surface discussed the previous section. Our definition matches with that in [3] if $\omega(\cdot, T) h(\cdot, T) \equiv 0$.

The following theorem gives us a microlocal characterization of the normal operator $\mathbf{L}_{\omega}^{*} \mathbf{L}_{\omega}$.

Theorem 3.9. The operator $\mathbf{L}_{\omega}^{*} \mathbf{L}_{\omega}$ is a pseudo-differential operator of order zero whose principal symbol is

$$
\sigma_{0}(x, \xi)=\frac{1}{4}\left(\int_{0}^{T} c^{2}\left(x_{+}(t)\right) \omega\left(x_{+}(t), t\right) d t+\int_{0}^{T} c^{2}\left(x_{-}(t)\right) \omega\left(x_{-}(t), t\right) d t\right) .
$$

Here, $x_{ \pm}(t)=r_{ \pm}(x, \xi)(t)$ is unit speed geodesic ray originated from $x$ at time $t=0$ along the direction of $\pm \xi$.

Proof. Let us consider the solution $q$ of the time reversed problem 3.14 with $h=\left.p\right|_{\mathrm{B} \times[0, T]}$. Applying the Duhamel's principle, we can write

$$
q(\cdot, t)=\int_{t}^{T} q(\cdot, t ; s) d s
$$

where $q(x, t ; s)$ satisfies

$$
\left\{\begin{array}{l}
c^{-2}(x) q_{t t}(x, t ; s)-\Delta q(x, t ; s)=0, \quad(x, t) \in \mathbb{R}^{d} \times(0, s), \\
q(x, s ; s)=0, \quad q_{t}(x, s ; s)=c^{2}(x) \omega(x, s) p(x, s), \quad x \in \mathbb{R}^{d} .
\end{array}\right.
$$

Therefore, denoting $q^{\prime}(\cdot, t ; s)=q_{t}(\cdot, t ; s)$,

$$
\mathbf{L}_{\omega}^{*}(p)=q_{t}(\cdot, 0)=\int_{0}^{T} q^{\prime}(\cdot, 0 ; s) d s-q(\cdot, 0 ; 0)=\int_{0}^{T} q^{\prime}(\cdot, 0 ; s) d s=\int_{0}^{T} \mathbf{L}(s)(p) d s .
$$

Let us show that $f \rightarrow \mathbf{L}(s)(p):=q^{\prime}(\cdot, 0 ; s)$ is a pseudo-differential operator with the principal symbol

$$
\sigma_{0}(x, \xi ; s)=\frac{1}{4}\left(c^{2}\left(x_{+}(s)\right) \omega\left(x_{+}(s), s\right)+c^{2}\left(x_{+}(s)\right) \omega\left(x_{-}(s), s\right)\right) .
$$

Indeed, we note that $q^{\prime}(x, t ; s)$ satisfies

$$
\left\{\begin{array}{l}
c^{-2}(x) q_{t t}^{\prime}(x, t ; s)-\Delta q^{\prime}(x, t ; s)=0, \quad(x, t) \in \mathbb{R}^{d} \times(0, s), \\
q^{\prime}(x, s ; s)=c^{2}(x) \omega(x, s) p(x, s), \quad q_{t}^{\prime}(x, s ; s)=0, \quad x \in \mathbb{R}^{d} .
\end{array}\right.
$$

Assume that $(x, \xi) \in\left(\mathbb{T}^{*} \Omega \backslash 0\right) \cap \mathrm{WF}(f)$ and consider the propagation of $p$, governed by the wave equation (1.1). The singularity of $f$ at $(x, \xi)$ is broken into two equal parts propagating along the geodesic rays $r_{ \pm}(x, \xi)$. Let us consider the propagation along $r_{+}(x, \xi)$. The projection of the propagated singularity at $t=s$ to $\mathbb{T}^{*} \mathbb{R}^{d}$ produces a corresponding singularity of $p(., s)$. Let us consider the propagation of that singularity due to the equation (3.15). Firstly, due to the end time condition at $t=s$, it is multiplied by $c^{2}(x) \omega(x(s), s)$. Then, it is broken into two equal parts propagating along two opposite directions (in reversed time). One of them hits back to $(x, \xi)$ at $t=0$ (this travel along $r_{+}(x, \xi)$ but in negative direction) and the other one lands 
outside of $\Omega$. Therefore, the strength of the recovered singularity at $(x, \xi)$, as just described, is $\frac{1}{2} c^{2}\left(x_{+}(s)\right) \omega\left(x_{+}(s), s\right)$ times that of the part of original singularity at $(x, \xi)$ propagating along the ray $r_{+}(x, \xi)$. Similar argument for the negative ray $r_{-}(x, \xi)$ gives us the second recovered singularity with the magnitude $\frac{1}{2} c^{2}\left(x_{+}(s)\right) \omega\left(x_{+}(s), s\right)$ of the part of original singularity at $(x, \xi)$ propagating along the negative ray $r_{-}(x, \xi)$. Since each part (propagating on each direction) is half of the original singularity, we obtain the recovered singularity is $\frac{1}{4}\left(c^{2}\left(x_{+}(s)\right) \omega\left(x_{+}(s), s\right)+\right.$ $\left.c^{2}\left(x_{-}(s)\right) \omega\left(x_{-}(s), s\right)\right)$ of the original singularity. This intuitively, shows that $\mathbf{L}(s) \mathbf{L}_{\omega}$ is a pseudodifferential operator of order zero with the principal symbol

$$
\frac{1}{4}\left(c^{2}\left(x_{+}(s)\right) \omega\left(x_{+}(s), s\right)+c^{2}\left(x_{-}(s)\right) \omega\left(x_{-}(s), s\right)\right) .
$$

A more rigorous proof can be done by writing the corresponding form of the parametrix for the wave equations (1.1) and 3.15. However, we skip it for the sake of simplicity.

Now, since $\mathbf{L}_{\omega}^{*} \mathbf{L}_{\omega}=\int_{0}^{T} \mathbf{L}(s) \mathbf{L}_{\omega} d s$, we obtain that $\mathbf{L}_{\omega}^{*} \mathbf{L}_{\omega}$ is a pseudo-differential operator of order zero with the symbol

$$
\frac{1}{4} \int_{0}^{T}\left(c^{2}\left(x_{+}(s)\right) \omega\left(x_{+}(s), s\right)+c^{2}\left(x_{-}(s)\right) \omega\left(x_{-}(s), s\right)\right) d s .
$$

This finishes the proof of the theorem.

Remark 3.10. Let us consider $\omega=\omega_{\epsilon}$ to be a family of smooth function that approximate the $\chi(x, t) \delta_{\partial \Omega}(x)$. Then, the setup for the observation on the surface $\partial \Omega$ is just the limit as $\epsilon \rightarrow 0$. We note that

$$
\lim _{\epsilon \rightarrow 0} \int_{0}^{T} c^{2}\left(x_{ \pm}(s)\right) \omega\left(x_{ \pm}(s), s\right) d s=\frac{c\left(x_{ \pm}\right) \chi\left(x_{ \pm}, t_{ \pm}\right)}{\cos \theta_{ \pm}} .
$$

Therefore,

$$
\lim _{\epsilon \rightarrow 0} \sigma_{0}(x, \xi)=\frac{1}{4}\left(\frac{c\left(x_{+}\right) \chi\left(x_{+}, t_{+}\right)}{\cos \left(\theta_{+}\right)}+\frac{c\left(x_{-}\right) \chi\left(x_{-}, t_{-}\right)}{\cos \left(\theta_{-}\right)}\right),
$$

which is the symbol $\mathbf{L}^{*} \mathbf{L}$ in Theorem 3.6.

\section{Numerical experiments}

In this section we implement the iterative methods presented in Section 2.1 for PAT for the observation on a surface $\partial \Omega$. We will employ the explicit formulation of $\mathbf{L}^{*}=\mathbf{L}_{0}^{*}$ presented in Section 3. We will chose the weight function $\chi$ to be independent of the time variable $t .7$ We note that under the visibility condition, Landweber's and the CG methods have linear convergence in $H_{0}\left(\Omega_{0}\right)=L^{2}\left(\Omega_{0}\right)$, since by Theorem 3.4 the inversion of $\mathbf{L} f=g$ is well-posed in this situation. If using $\mathbf{L}^{*}=\mathbf{L}_{1}^{*}$, we would obtain the linear rate of convergence in $\mathbb{X}_{1} \simeq H^{1}\left(\Omega_{0}\right)$. However, we will refrain from that choice.

We only consider two-dimensional simulations and assume $\Omega$ to be a disc centered at the origin: $\Omega=B_{R}(0)=\left\{x \in \mathbb{R}^{2}:|x|<R\right\}$. All presented results assume non-constant sound speed. We consider the following test cases

\footnotetext{
${ }^{7}$ Other choices of $\chi$ may result in better conditioning of the problem. However, studying optimal preconditioning is beyond the scope of this article.
} 
(T1) Complete data;

(T2) Partial data, visible phantom (i.e., the visibility condition holds);

(T3) Partial data, invisible phantom (i.e., the invisibility condition holds);

We will compare the results for the Landweber's method, Nesterov's method, the CG method (as proposed in the present paper) as well as the iterative time reversal algorithm proposed in [55]. Thereby we investigate the numerical speed of convergence as well as stability and accuracy of all these algorithms. For Landweber's and Nesterov's method we have taken the step size equal to $\gamma=1$, which worked well in all our numerical simulations.

As described in Subsection 4.1 the proposed iterative schemes are implemented by numerical realizations of all involved operators. Thereby the most crucial steps are accurate discrete solvers for the forward and backward wave equation. For that purpose we implemented the $k$-space method (described in Appendix B) that is an efficient FFT based numerical solution method that does not suffer from numerical dispersion that arises when solving the wave equation with standard finite difference or finite element methods. We note that to compute the adjoint operator $\mathbf{L}^{*}$ numerically, we make use of the formulation (3.5).
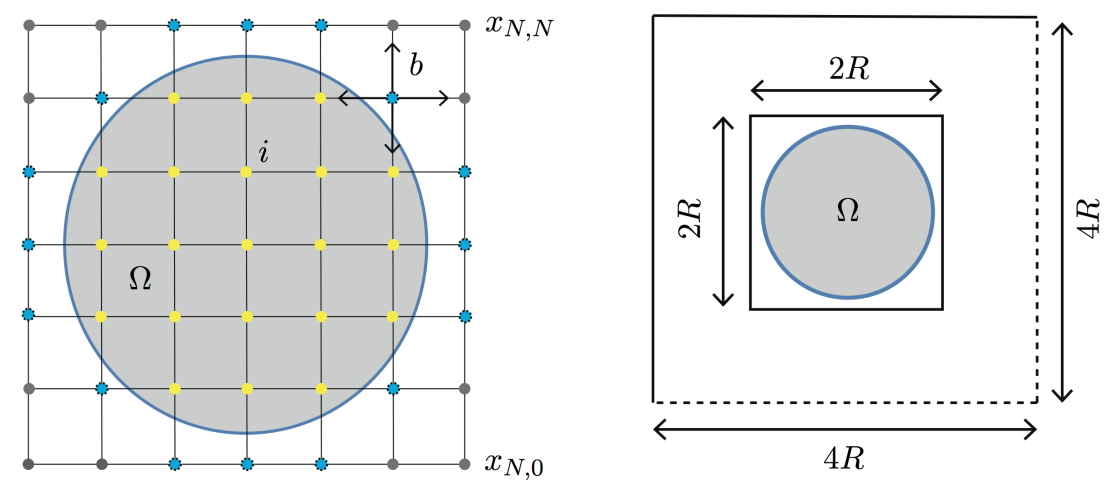

Figure 4.1: Left: The discrete domain $\Omega_{N}$ is defined as the set of all indices $i \in\{0, \ldots, N\}^{2}$ with $x_{i} \in \Omega$. The index $b$ is contained in the discrete boundary $\partial \Omega_{N}$, because one of its neighbors is contained in $\Omega_{N}$. Right: The domain $[-R, R]$ is embedded in a larger domain $[-2 R, 2 R]$ to avoid effects due to periodization.

\subsection{Numerical realization}

The iterative approaches for solving the equation $\mathbf{L} f=g$ are implemented with discrete counterparts of all operators introduced above. Thereby the function $f: \mathbb{R}^{2} \rightarrow \mathbb{R}$ is represented by a discrete vector

$$
\mathbf{f}=\left(f\left(x_{i}\right)\right)_{i_{1}, i_{2}=0}^{N} \in \mathbb{R}^{(N+1) \times(N+1)},
$$

where $x_{i}=(-R,-R)+2 i R / N$ for $i=\left(i_{1}, i_{2}\right) \in\{0, \ldots, N\}^{2}$ are equidistant grid points in the square $[-R, R]^{2}$. We define the discrete domain $\Omega_{N} \subset\{0, \ldots, N\}^{2}$ as the set of all indices $i$ with $x_{i} \in \Omega$. Further, the discrete measurements are made on parts of the discrete boundary $\partial \Omega_{N}$, that is defined as the set of all elements $b=\left(b_{1}, b_{2}\right) \in\{0, \ldots, N\}^{2} \backslash \Omega_{N}$ for which at least 
one of the discrete neighbors $\left(b_{1}+1, b_{2}\right),\left(b_{1}-1, b_{2}\right),\left(b_{1}, b_{2}+1\right),\left(b_{1}, b_{2}-1\right)$ is contained in $\Omega_{N}$, see the right image in Figure 4.1. All phantoms in our numerical simulations are chosen to have support in a compact subset $\Omega_{0}$ of $\Omega$. We will choose the discrete version of $\Omega_{0}$ to be the set $\left\{x_{i}: i \in \Omega_{N}\right\}$.

The discrete forward operator can be written in the form

$$
\mathrm{L}_{N, M}: \mathbb{R}^{(N+1) \times(N+1)} \rightarrow \mathbb{R}^{\left|\partial \Omega_{N}\right| \times(M+1)}: \mathrm{f} \mapsto\left(\mathrm{R}_{N, M} \circ \mathrm{W}_{N, M}\right) \mathrm{f} .
$$

Here $M+1$ is the number of equidistant temporal sampling points in $[0, T], \mathrm{W}_{N, M}$ is a discretization of the solution operator for the wave equation and $\mathrm{R}_{N, M}$ the linear operator that restricts the discrete pressure to spatial grid points restricted to $\partial \Omega_{N} \subset\{0, \ldots, N\}^{2}$. The adjoint operator is then given by $\mathrm{L}_{N, M}=\mathrm{D}_{0} \circ \mathrm{W}_{N, M}^{*} \circ \mathrm{R}_{N, M}^{*}$, where $\mathrm{R}_{N, M}^{*}$ is the embedding operator from

$\mathbb{R}^{\left|\partial \Omega_{N}\right|}$ to $\mathbb{R}^{(N+1) \times(N+1)}, \mathrm{W}_{N, M}^{*}$ is the solution operator the adjoint wave equation $(\mathrm{B} .7)$, and $\mathrm{D}_{0}$ a discretization of the time derivative evaluated at $t=0$ and restricted to $\Omega_{0}$.

For computing the solution operator $\mathrm{W}_{N, M}$ we use the $k$-space method described in Appendix $\mathrm{B}$, In the actual implementation of Algorithm 1, the Fourier transform of the function $f$ is replaced by the FFT algorithm applied to $f$ (and likewise for the inverse Fourier transform). When applied directly to the given function values, the FFT algorithm causes the numerical solution to be $2 R$ periodic. For the numerical solution of the wave equation we therefore embed the data vector $\mathrm{f} \in \mathbb{R}^{(N+1) \times(N+1)}$ in a larger vector in $\mathbb{R}^{(2 N+1) \times(2 N+1)}$, whose entries correspond to sampled values on an equidistant grid in $[-2 R, 2 R]^{2}$ (see the right image in Figure 4.1 ). As the sound speed is assumed to be equal to one outside of $\Omega$, the numerical solution for times $t \leq 2 R$ (which will always be the case in our simulations) is free from periodization artifacts in the domain $\Omega$ and on the measurement surface.

Remark 4.1 (Numerical complexity of $k$-space based iterative algorithms). Using the FFT algorithm any time step in the $k$-space method (summarized in Algorithm 1) can be implemented using $\mathcal{O}\left(N^{2} \log N\right)$ floating point operations (FLOPS). Performing $M \sim N$ time steps therefore yields to $\mathcal{O}\left(N^{3} \log N\right)$ algorithms for implementing the forward operator $\mathrm{L}_{N, M}$ and its adjoint $\mathrm{L}_{N, M}^{*}$. Consequently, performing one iterative step (for example using the CG or the Landweber iteration) is almost as fast as applying the filtered backprojection type algorithm (which requires $\mathcal{O}\left(N^{3}\right)$ FLOPS) for evaluating the adjoint or the inverse of $\mathbf{L}$. In three spatial dimensions the complexity of the $k$-space method scales to $\mathcal{O}\left(N^{4} \log N\right)$. In this case one iterative step is already faster than filtered backprojection type algorithms (which in this case requires $\mathcal{O}\left(N^{5}\right)$ FLOPS). As we will see in the numerical results presented below, around 10 iterations with the CG method already gives very accurate reconstruction results. This shows that our iterative algorithms are a good option for PAT image reconstruction even in situations, where an explicit filtered backprojection type formula is available.

\subsection{Test case (T1): Complete data}

We consider the nontrapping sound speed (taken from [55])

$$
c(x)=1+w(x)\left(0.1 \cos \left(2 \pi x_{1}\right)+0.05 \sin \left(2 \pi x_{2}\right)\right),
$$



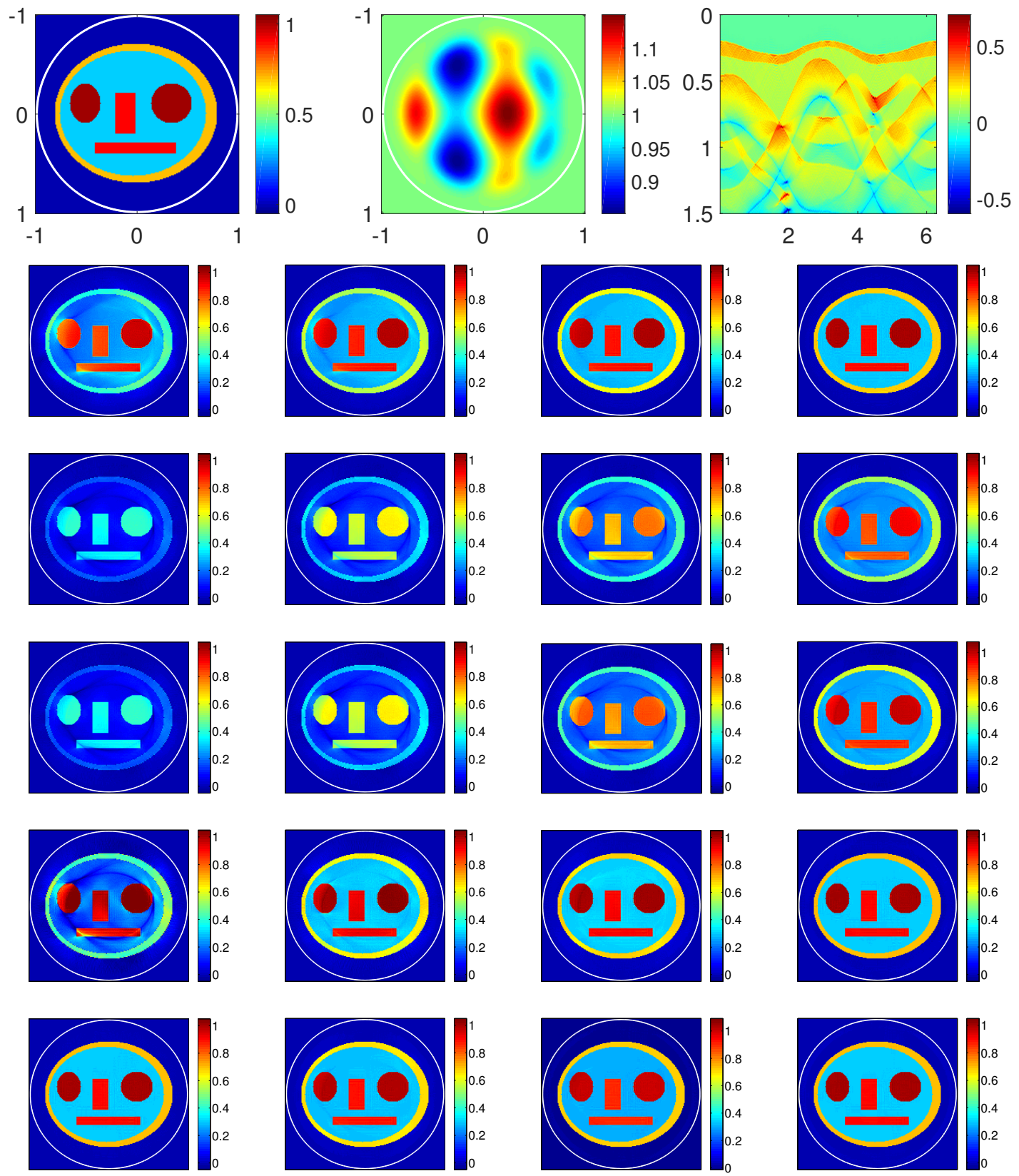

Figure 4.2: Test Case (T1), Reconstructions from exact Data. Row 1: Initial pressure data $f$ (left), non-trapping sound speed $c$ (middle), and pressure data $\mathbf{L} f$ (right). The white dots indicate the measurement curve. Row 2: Iterative time reversal (after 1, 2, 3 and 5 iterations). Row 3: Landweber's method (after 1, 2, 3 and 5 iterations). Row 4: Nesterov's method (after 1, 2, 3 and 5 iterations). Row 5: CG method (after 1, 2, 3 and 5 iterations). Row 6: Results after 10 iterations using iterative time reversal, Landweber's method, Nesterov's method, CG method (from left to right). 
where $w: \mathbb{R}^{2} \rightarrow[0,1]$ is a smooth function that vanishes outside $B_{1}(0)$ and is equal to one on $B_{1 / 2}(0)$. The sound speed $c$, the phantom $f$ and the corresponding full data $\mathbf{L} f$ are illustrated in the top row in Figure 4.2. For the results presented in this section we use $R=1$ and $N=200$, which yields a spatial step size of $h_{x}=2 R / N=1 / 100$. We further use a final time $T=1.5$ and take $M=800$ for the temporal discretization.
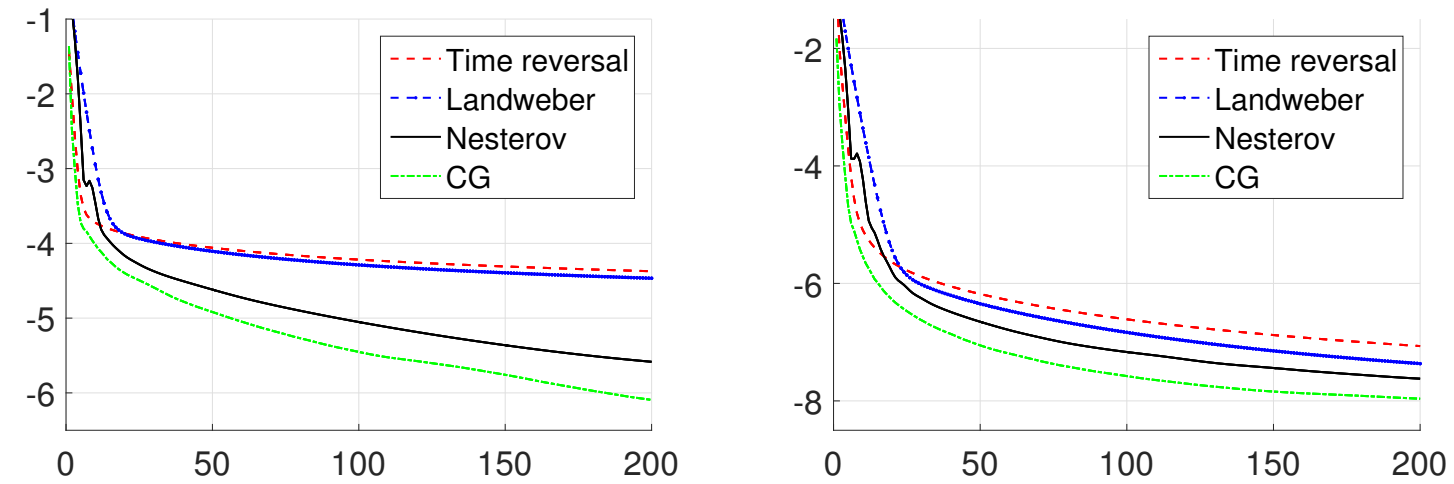

Figure 4.3: TEST CASE (T1), CONVERGENCE BEHAVIOR FOR EXACT DATA Left: Logarithm of squared reconstruction error $\left\|\mathrm{f}_{n}-\mathbf{f}\right\|_{2}^{2}$ in dependence of the iteration number. Right: Logarithm of residual $\left\|\mathrm{L}_{N, M} \mathrm{f}_{n}-\mathrm{g}\right\|_{2}^{2}$ in dependence of the iteration number.

We performed iterative reconstructions using the following methods:

(a) Iterative time reversal method

(b) Landweber's method

(c) Nesterov's method

(d) CG method.

Figure 4.2 shows reconstruction results using these methods after 1, 2, 3, 5, and 10 iterations. One notices that all iterations converge quite rapidly to the phantom to be recovered. In the initial iterations the time reversal and the $\mathrm{CG}$ method produce the best results. After 10 iterations all reconstructions look very similar to the initial phantom. To investigate the convergence behavior further, in Figure 4.3 we plot the logarithm of the squared discrete $L^{2}$ reconstruction error and squared residual

$$
\begin{aligned}
\left\|\mathbf{f}_{n}-\mathbf{f}\right\|_{2}^{2} & :=\sum_{i}\left|\mathbf{f}_{n}[i]-\mathbf{f}[i]\right|^{2} h_{x}^{2} \simeq\left\|f-f_{n}\right\|_{L^{2}}^{2}, \\
\left\|\mathrm{~L}_{N, M} \mathbf{f}_{n}-\mathbf{g}\right\|^{2} & :=\sum_{b, j}\left|\mathrm{~L}_{N, M} \mathbf{f}_{n}[b, j]-\mathrm{g}[b, j]\right|^{2} h_{x} h_{t} \simeq\left\|\mathbf{L} f_{n}-g\right\|_{L^{2}}^{2},
\end{aligned}
$$

respectively. One concludes from Figure 4.3 , that all iterative schemes converge quite rapidly. In particular the CG method is the fastest.

In order to further investigate the behavior of the algorithms we repeated the computations with inexact data. To that end, we generated the data on a different grid, where we use $N=350$ 
and $M=1300$ (recall that the iterative algorithm uses $N=200$ and $M=800$ ). Further, we added Gaussian white noise to the data with a standard deviation equal to $5 \%$ of the $L^{2}$-norm of $\mathrm{L}_{N, M} \mathrm{f}$. The total $L^{2}$-error in the data is 0.049 and the $L^{2}$-norm of the exact data is $\|\mathrm{g}\|_{2}=0.44$.

Figure 4.4 shows the reconstruction results from inexact data using iterative time reversal, Landweber's, Nesterov's, and the CG methods. The errors and the residuals again decrease quite rapidly in the first iterative steps. However after about 10 iterations the error as well as the residuals do not further decrease. Consequently, the iterations can be stopped at a certain iteration index $n_{\star}$. This is due to the noise in the data which causes the data to be outside the range of $\mathrm{L}_{N, M}$. However, these results also reveal that we are in a stable situation, because the error does not significantly increase after reaching the stopping index $n_{\star}$.
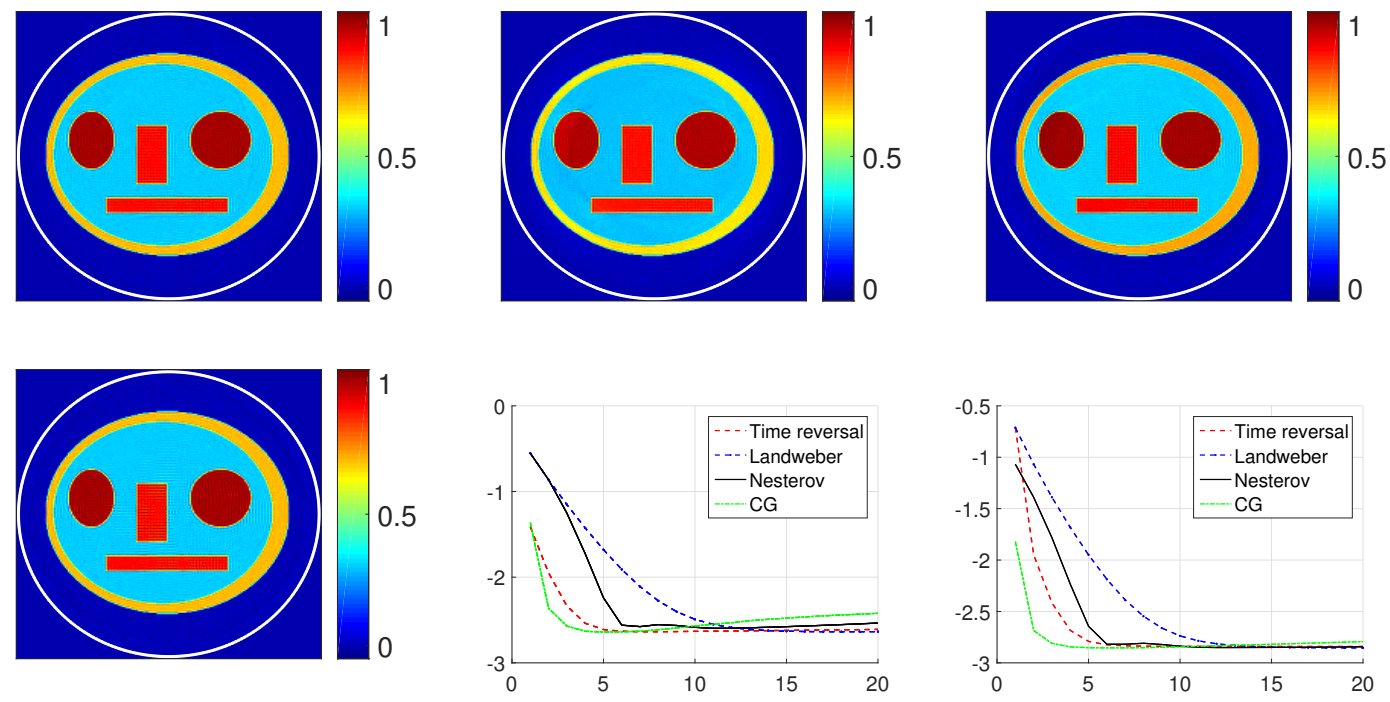

Figure 4.4: TeSt CASE (T1), InEXACT DATA. Top left: Iterative time reversal, Top center: Landweber's method, top right: Nesterov's method. Bottom left: the CG method (all after 10 iterations). Bottom center: squared error. Bottom right: squared residuals.
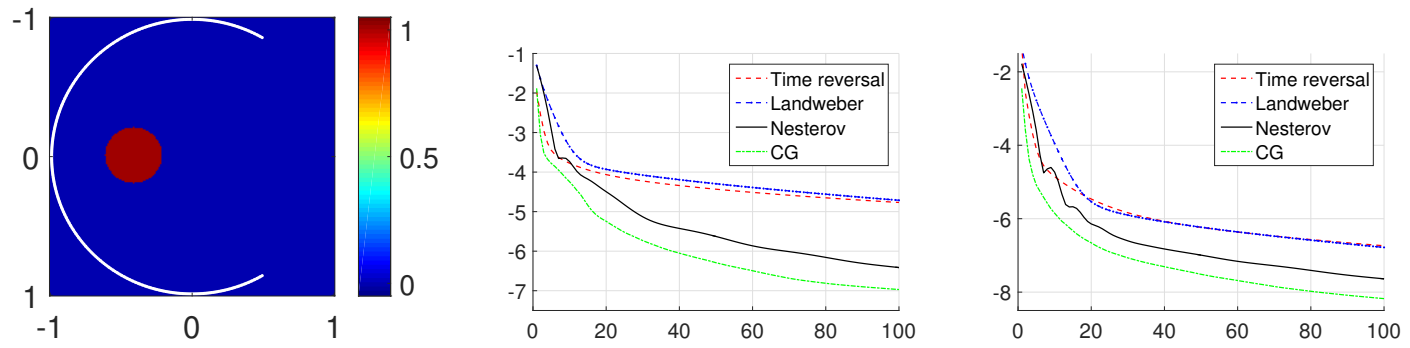

Figure 4.5: TEST CASE (T2), EXACT DATA. Left: Initial pressure data $f$. Middle: Logarithm of squared reconstruction error $\left\|\mathbf{f}_{n}-\mathbf{f}\right\|_{2}^{2}$ in dependence of the iteration number. Right: Logarithm of residual $\left\|\mathrm{L}_{N, M} \mathrm{f}_{n}-\mathrm{g}\right\|_{2}^{2}$ in dependence of the iteration number.

Note that we do not show results using the Landweber's method proposed in [7]. Due to the smoothing operator $-\Delta^{-1}$ (which is the adjoint of the embedding $H_{0}^{1}(\Omega) \hookrightarrow L^{2}(\Omega)$ ), that 

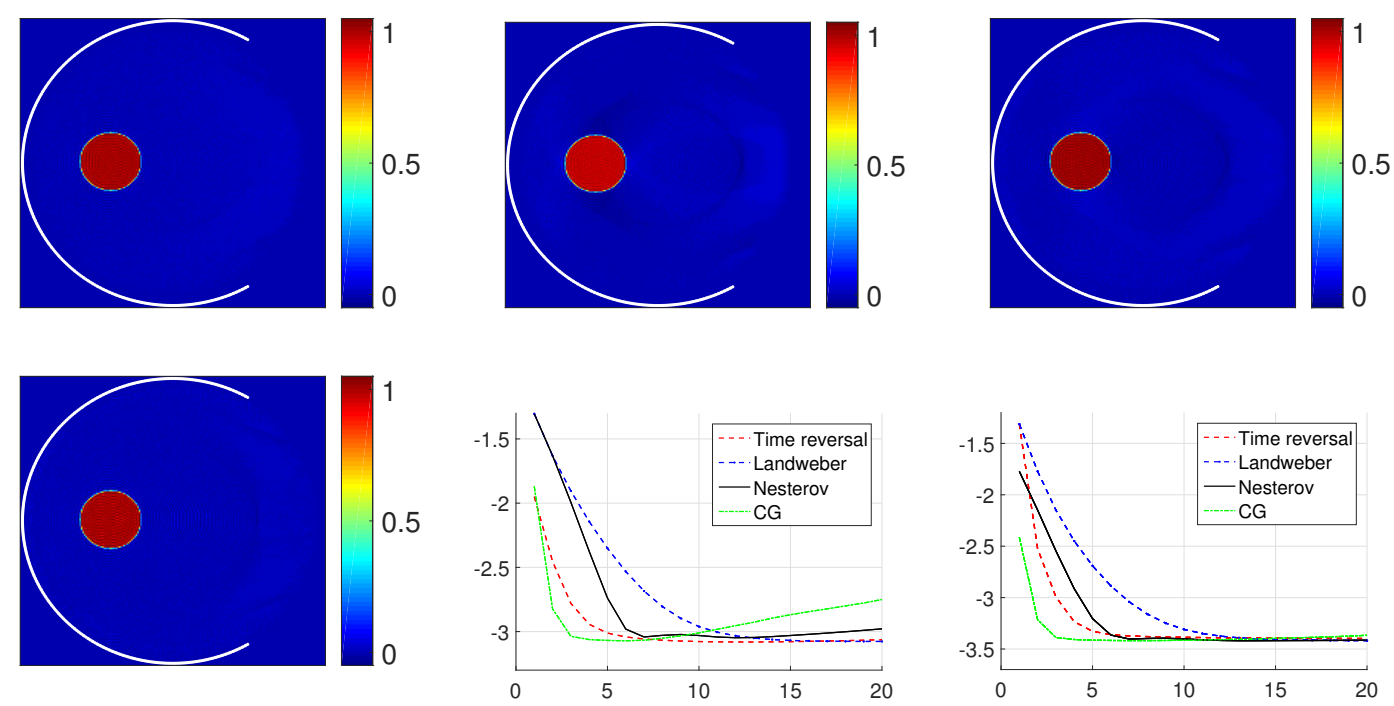

Figure 4.6: TEST CASE (T2), InEXACT DATA. Top left: Iterative time reversal, Top center: Landweber's method, top right: Nesterov's method. Bottom left: the CG method (all after 10 iterations). Bottom center: squared error. Bottom right: squared residuals.

method is much slower than the Landweber's method presented in the present article. On the other hand, the application of $-\Delta^{-1}$ may have the advantage of stabilizing the iteration.

\subsection{Test case (T2): Partial data, visible phantom}

As next test case we investigate the case of partial data where all singularities of the phantom are visible. As before we compare iterative time reversal, Landweber's, Nesterov's, and the CG methods using the sound speed given in (4.2). We again take $N=200, R=1, T=1.5$ and $M=800$. The the phantom is shown in shown left image in Figure 4.5. One notices that the partial data have been collected on an arc with opening angle $4 \pi / 3$. The middle and right image in Figure 4.5 show the reconstruction error and the residuals depending on the iteration index $n$. One observes a similar asymptotic behavior as for the test case (T1) with exact data. In particular the $\mathrm{CG}$ iteration is again the most rapidly converging method. Also the convergence behavior in the first iterations is similar to the complete data case; due to space limitations we do not show the corresponding pictures.

To avoid inverse crimes and to investigate the behavior of the algorithms under real life scenario, we repeated the simulations with inexact data where we simulated the data on a different grid (using $N=350$ and $M=1300$ ) and further added Gaussian noise to the data (again with a standard deviation equal to $5 \%$ of the $L^{2}$-norm of the exact data). The reconstruction results from inexact data are shown in Figure 4.6. They clearly demonstrate that all schemes provide good results. The CG method is again the fastest. The error $\left\|\mathrm{L}_{N, M} \mathrm{f}-\mathrm{g}^{\delta}\right\|_{2}$ in the data is 0.0258. The residuals after 10 iterations are 0.2208 for the iterative time reversal, 0.0196 for the CG, 0.0199 for Nesterov's, and 0.0222 for the Landweber's methods. This in particular also shows that the discrepancy principle yields a well defined stopping index with an reconstruction 
error in the order of the data error.
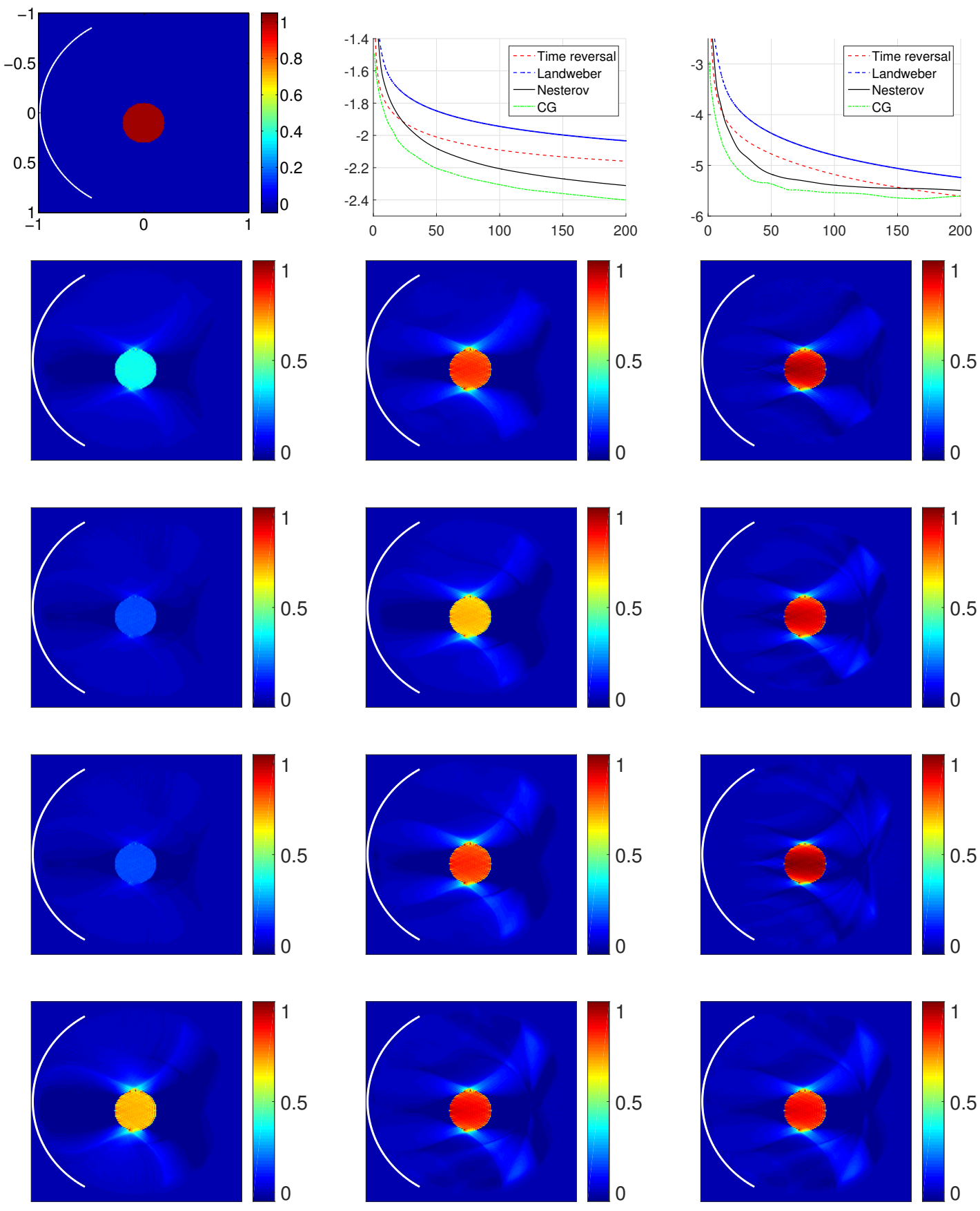

Figure 4.7: Test CASe (T3): PARTial DATA, invisible Phantom Row 1: Initial pressure data $f$ (left), Logarithm of squared reconstruction error $\left\|\mathbf{f}_{n}-\mathbf{f}\right\|_{2}^{2}$ (middle), and logarithm of residual $\left\|\mathrm{L}_{N, M} \mathrm{f}_{n}-\mathrm{g}\right\|_{2}^{2}$ (right). Row 2: Iterative time reversal (after 1, 10 and 200 iterations). Row 3: Landweber's method (after 1, 10 and 200 iterations). Row 4: Nesterov's method (after 1, 10 and 200 iterations). Row 5: the CG method (after 1, 10 and 200 iterations). 

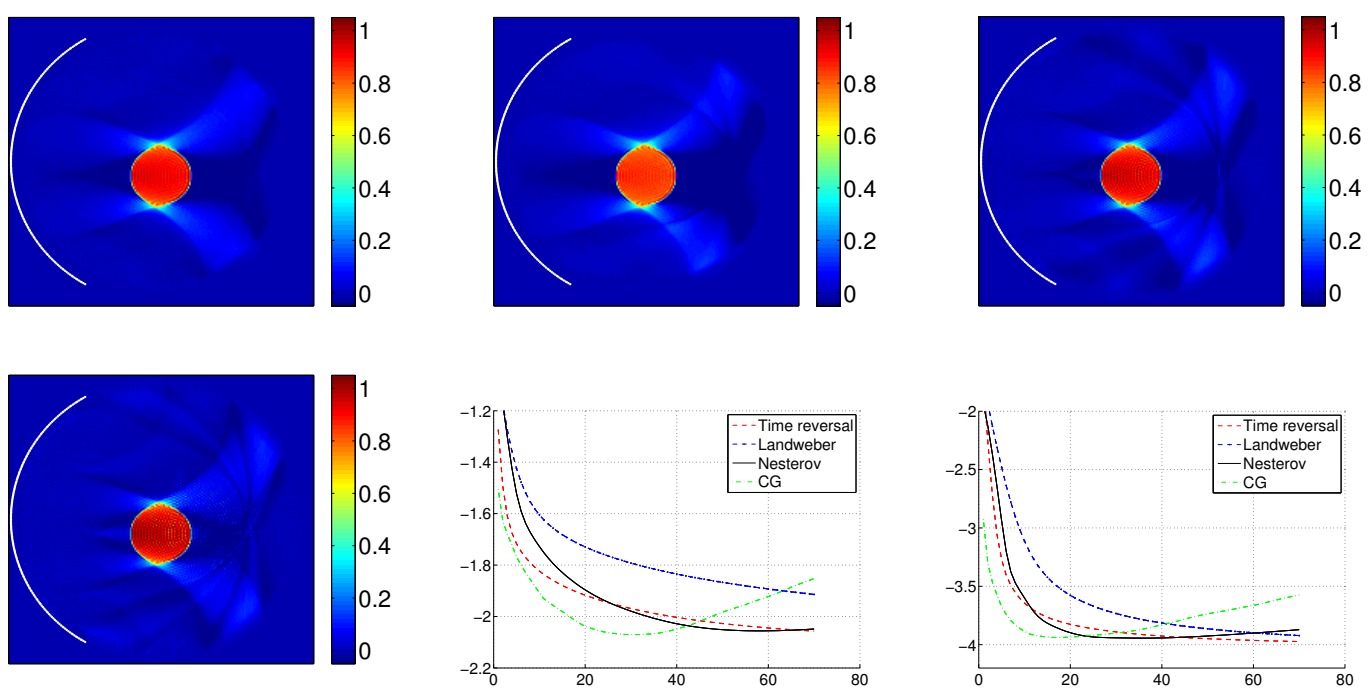

Figure 4.8: TEST CASE (T3), InEXACT DATA. Top left: Iterative time reversal, Top center: Landweber's method, top right: Nesterov's method. Bottom left: the CG method (all after 30 iterations). Bottom center: squared error. Bottom right: squared residuals.

\subsection{Test case (T3): Partial data, invisible phantom}

In this case we investigate the ill-posed problem, since the invisibility condition holds. We use again the sound speed given in 4.2 and take $N=200, R=1, T=1.5$ and $M=800$. The phantom is shown in the top left image in Figure 4.7. The partial data are collected on an arc with opening angle $2 \pi / 3$ (the measurement curve). The invisibility condition holds in this setup. Rows 2 to 5 in Figure 4.7 show reconstruction results with iterative time reversal, Landweber's, Nesterov's, and the CG methods after 1, 10 and 200 iterations. Because the inverse problem is ill-posed no convergence rate results for the Landweber, Nesterov's, and CG methods are available. And indeed one observes that the reconstruction results are worse compared to the the case of a completely visible phantom. Again we investigated the convergence behavior more carefully. For that purpose in the top row of 4.7 we again shows the reconstruction error and the residuals depending on the iteration index $n$. While the residuals tend to zero quite fast, reconstruction error now decreases much slower than in the previous examples. Nevertheless also in this situation the CG iteration clearly yields the smallest reconstruction error for a given number of iterations.

Again we repeated the simulations with inexact data where we simulated the data on a different grid and further added Gaussian noise to the data. Due the ill-posedness of the problem we cannot expect complete convergence for noisy data. In fact, as can be seen in Figure 4.8 all iterations show the typical semi-convergence behavior: The error decreases until a certain optimal index $n^{\star}$, after which the error starts to increase. Stopping the iteration (for example with Morozovs discrepancy principle) yields approximate but stable solutions. Incorporating additional regularization could further improve the results. Such investigations, however, are beyond the scope of this paper. 


\section{Conclusion and outlook}

In this paper we derived, analyzed, and implemented iterative algorithms for PAT with variable sound speed. We considered the full and partial data situation. In most of the cases under consideration, Landweber's method performs almost as well as the iterative time reversal method while Nesterov's and CG method converge faster. Note that the semi-convergence of the CG method is visible for all noisy data. The Landweber's and the iterative time reversal methods are asymptotically much slower and therefore also the semi-convergence phenomenon appears later. This is also the case for the full data problem; however full data seems to further stabilize the problem above the visible data problem. Especially Landweber's and Nesterov's methods are convenient for regularization, which we will investigate in an upcoming work.

\section{Acknowledgement}

Linh Nguyen's research is partially supported by the NSF grants DMS 1212125 and DMS 1616904. He also thanks the department of Mathematics of the University of Innsbruck for financial support and hospitality during his visit in 2016. The authors are thankful to the anonymous referees for various helpful comments and suggestions.

\section{A Formulation of weak solution}

In this section, we define the (weak) solution $q(x, t)$ in (3.1). To that end, we follow [7]. Let us denote by $V$ the Hilbert space with the inner product

$$
\left\langle\varphi_{1}, \varphi_{2}\right\rangle=\int_{\Omega} \varphi_{1}(x) \varphi_{2}(x)+\int_{\mathbb{R}^{n}} \nabla \varphi_{1}(x) \nabla \varphi_{2}(x) d x,
$$

and $V^{\prime}$ the dual space of $V$. Then, space of test functions $C_{0}^{\infty}\left(\mathbb{R}^{n}\right)$ is dense in $V$. Therefore, $V^{\prime}$ is a subset of the space of distribution $\mathcal{D}^{\prime}\left(\mathbb{R}^{n}\right)$. Moreover,

$$
V^{\prime}=\left\{d \in \mathcal{D}^{\prime}\left(\mathbb{R}^{n}\right): \text { there is } C>0 \text { such that: }|(d, f)| \leq C\|f\|_{V}, \text { for all } f \in C_{0}^{\infty}\left(\mathbb{R}^{n}\right)\right\} .
$$

Here and elsewhere, $(d, f)$ is the action of the distribution $d$ on the test function $f$.

Definition A.1. Let $h \in C^{\infty}(\partial \Omega \times(0, T))$. A weak solution of the problem

$$
\left\{\begin{array}{l}
c^{-2}(x) z_{t t}(x, t)-\Delta z(x, t)=0, \quad(x, t) \in\left(\mathbb{R}^{d} \backslash \partial \Omega\right) \times(0, T), \\
z(x, T)=0, \quad z_{t}(x, T)=0, \quad x \in \mathbb{R}^{d}, \\
{[z](y, t)=0,\left[\frac{\partial z}{\partial \nu}\right](y, t)=h(y, t), \quad(y, t) \in \partial \Omega \times[0, T] .}
\end{array}\right.
$$

is a function $z$ that satisfies:

1. $z \in L^{2}(0, T ; V), z^{\prime} \in L^{2}\left(0, T ; L^{2}\left(\mathbb{R}^{n}\right)\right), z^{\prime \prime} \in L^{2}\left(0, T ; V^{\prime}\right)$,

2. $z(T)=z^{\prime}(T)=0$, 
3. for any $v \in L^{2}(0, T ; V)$, we have

$$
\begin{array}{r}
\int_{0}^{T}\left\langle c^{-2}(\cdot) z_{t t}(\cdot, t), v(\cdot, t)\right\rangle_{\left(V^{\prime}, V\right)} d t+\int_{0}^{T} \int_{\mathbb{R}^{d}} \nabla z(x, t) \nabla v(x, t) d x d t= \\
-\int_{0}^{T} \int_{\partial \Omega} h(y, t) v(y, t) d y d t .
\end{array}
$$

Its existence and uniqueness can be found in [7].

Assume that $v \in C^{\infty}\left(\mathbb{R}^{d} \times \overline{\mathbb{R}}\right)$ such that $v(\cdot, t) \in C_{0}^{\infty}\left(\mathbb{R}^{d}\right)$ for all $t \in \overline{\mathbb{R}}$. Taking integration by parts of the second term of the left hand side with respect to $x$, we can write A.2 in the form

$$
\int_{0}^{T}\left(c^{-2} z_{t t}(\cdot, t)-\Delta z(\cdot, t), v(\cdot, t)\right) d t=-\int_{0}^{T} \int_{\partial \Omega} h(y, t) v(y, t) d y d t
$$

or

$$
c^{-2}(\cdot) z_{t t}(\cdot, t)-\Delta z(\cdot, t)=-\delta_{\partial \Omega}(\cdot) h(\cdot, t)
$$

Therefore, A.1 can be formally rewritten as the nonhomogeneous wave problem

$$
\left\{\begin{array}{l}
c^{-2}(x) z_{t t}(x, t)-\Delta z(x, t)=-\delta_{\partial \Omega}(x) h(x, t), \quad(x, t) \in \mathbb{R}^{d} \times(0, T), \\
z(x, T)=0, \quad z_{t}(x, T)=0, \quad x \in \mathbb{R}^{d} .
\end{array}\right.
$$

\section{B The $k$-space method for numerically solving the wave equa- tion}

In this subsection we briefly describe the $k$-space method as we use it to numerically compute the solution of wave equation, which is required for evaluating the forward operator $\mathbf{L}$ and its adjoint $\mathbf{L}^{*}$.

Consider the solution $p: \mathbb{R}^{2} \times(0, T) \rightarrow \mathbb{R}$ of the two-dimensional wave equation

$$
\begin{array}{ll}
c^{-2}(x) p_{t t}(x, t)-\Delta p(x, t)=s(x, t) & \text { for }(x, t) \in \mathbb{R}^{2} \times(0, T), \\
p(x, 0)=f(x) & \text { for } x \in \mathbb{R}^{2}, \\
p_{t}(x, 0)=0 & \text { for } x \in \mathbb{R}^{2},
\end{array}
$$

where $s: \mathbb{R}^{2} \times(0, T) \rightarrow \mathbb{R}$ is a given source term and $f: \mathbb{R}^{2} \rightarrow \mathbb{R}$ the given initial pressure. Several well investigated methods for numerically solving (B.1) (and analogously for the wave equation in higher dimensions) are available and have been used for photoacoustic tomography. This includes finite difference methods [10, 52, 59], finite element methods [7] as well as Fourier spectral and $k$-space methods [12, 33, 62]. In this paper we use a $k$-space method for numerically solving (B.1) because this method does not suffer from numerical dispersion. The $k$-space method is implemented in the freely available $k$-wave toolbox (see [62]); in order to be flexible in our implementations we have developed our own code as described below.

The $k$-space method makes the ansatz (see [12, 43, 60])

$$
p(x, t)=w(x, t)-v(x, t) \quad \text { for }(x, t) \in \mathbb{R}^{2} \times(0, T),
$$


where the pressure $p$ is written as linear combination of the auxiliary quantities $w:=c_{0}^{2} / c^{2} p$ and $v:=\left(1-c_{0}^{2} / c^{2}\right) p$. Here $c_{0}>0$ a suitable constant; we take $c_{0}:=\max \left\{c(x): x \in \mathbb{R}^{2}\right\}$. One easily verifies that the wave (B.1) is equivalent to the following system of equations,

$$
\left\{\begin{aligned}
w_{t t}(x, t)-c_{0}^{2} \Delta w(x, t) & =c_{0}^{2} s(x, t)-c_{0}^{2} \Delta v(x, t) & & \text { for }(x, t) \in \mathbb{R}^{2} \times(0, T), \\
v(x, t) & =\frac{c(x)^{2}-c_{0}^{2}}{c_{0}^{2}} w(x, t) & & \text { for }(x, t) \in \mathbb{R}^{2} \times(0, T) .
\end{aligned}\right.
$$

Interpreting $\Delta v$ as an additional source term, the first equation in (B.5) is a standard wave equation with constant sound speed. This suggests the time stepping formula (see [12, 43])

$$
\begin{aligned}
& w\left(x, t+h_{t}\right)=2 w(x, t)-w\left(x, t-h_{t}\right) \\
& \quad-4 \mathcal{F}_{\xi}^{-1}\left[\sin \left(c_{0}|\xi| h_{t} / 2\right)^{2} \mathcal{F}_{x}[w(x, t)-v(x, t)]-\left(c_{0} h_{t} / 2\right)^{2} \operatorname{sinc}\left(c_{0}|\xi| h_{t} / 2\right)^{2} \mathcal{F}_{x}[s(x, t)]\right],
\end{aligned}
$$

where $\mathcal{F}_{x}$ and $\mathcal{F}_{\xi}^{-1}$ denote the Fourier and inverse Fourier transform in the spatial variable $x$ and the spatial frequency variable $\xi$, respectively, and $h_{t}>0$ is a time stepping size. Note that the factor $4 \operatorname{sinc}\left(c_{0}|\xi| h_{t} / 2\right)^{2}$ is a distinctive feature of the $k$-space method and replaces the factor $\left(c_{0}|\xi| h_{t}\right)^{2}$ arising in standard finite differences. For constant sound speed we have $v=0$, in which case the solution of equation (B.1) exactly satisfies (B.6) (see, e.g., [12]). In the case of variable sound speed there is no such equivalence because $v$ is itself dependent on $w$. Nevertheless, in any case (B.6) serves as the basis of an efficient and accurate iterative time stepping scheme for numerically computing the solution of the wave equation.

The resulting $k$-space method for solving (B.1) is summarized in Algorithm 1 .

Algorithm 1 (The $k$-space method). For given initial pressure $f(x)$ and source term $s(x, t)$ approximate the solution $p(x, t)$ of (B.1) as follows:

(1) Define initial conditions $w\left(x,-h_{t}\right)=w(x, 0)=v(x, 0)=c_{0}^{2} / c^{2} f(x)$;

(2) Set $t=0$;

(3) Compute $w\left(x, t+h_{t}\right)$ by evaluating (B.6);

(4) Compute $v\left(x, t+h_{t}\right):=\left(c^{2}(x) / c_{0}^{2}-1\right) w\left(x, t+h_{t}\right)$;

(5) Compute $p\left(x, t+h_{t}\right):=w\left(x, t+h_{t}\right)-v\left(x, t+h_{t}\right)$;

(6) Set $t \leftarrow t+h_{t}$ and go back to (3).

Algorithm 1 can directly be used to evaluate the forward operator $\mathbf{L} f$ by taking $s(x, t)=0$ and restricting the solution to the measurement surface $S_{R}$, that is $\mathbf{L} f=\left.p\right|_{S_{r} \times(0, T)}$. Recall that the adjoint operator is given by $\mathbf{L}^{*} g=q_{t}(\cdot, 0)$, where $q: \mathbb{R}^{2} \times(0, T) \rightarrow \mathbb{R}$ satisfies the adjoint wave equation

$$
\begin{array}{ll}
c^{-2}(x) q_{t t}(x, t)-\Delta q(x, t)=-\delta_{S_{R}}(x) g(x, t) & \text { for }(x, t) \in \mathbb{R}^{2} \times(0, T) \\
q_{t}(x, T)=q(x, T)=0 & \text { for } x \in \mathbb{R}^{2} .
\end{array}
$$

By substituting $t \leftarrow T-t$ and taking $s(x, t)=g(x, T-t) \delta_{S}(x)$ as source term in (B.1), Algorithm 1 can also be used to evaluate the $\mathbf{L}^{*}$. In the partial data case where measurements 
are made on a subset $S \subsetneq S_{R}$ only, the adjoint can be implemented by taking the source $s(x, t)=\chi(x, t) g(x, T-t) \delta_{S_{R}}(x)$ with an appropriate window function $\chi(x, t)$. In order to use all available data, in our implementations we take the window function to be equal to one on the observation part $S$ and zero outside. This choice of the window function is known to create streak artifacts into the picture [21, 51, 5. However, the artifacts fade away quickly after several iterations when the problem is well-posed.

\section{References}

[1] S. Acosta And C. Montalto, Multiwave imaging in an enclosure with variable wave speed, Inverse Problems, 31 (2015), p. 065009.

[2] M. Agranovsky and P. Kuchment, Uniqueness of reconstruction and an inversion procedure for thermoacoustic and photoacoustic tomography with variable sound speed, Inverse Problems, 23 (2007), p. 2089.

[3] S. R. Arridge, M. M. Betcke, B. T. Cox, F. Lucka, and B. E. Treeby, On the adjoint operator in photoacoustic tomography, Inverse Problems, 32 (2016), p. 115012 (19pp).

[4] O. Axelsson And J. Karátson, On the rate of convergence of the conjugate gradient method for linear operators in Hilbert space, Numerische Mathematik, 48 (2002), pp. 499523.

[5] L. L. Barannyk, J. Frikel, and L. V. Nguyen, On Artifacts in Limited Data Spherical Radon Transform: Curved Observation Surface, Inverse Problems, 32 (2015).

[6] A. Beck And M. Teboulle, A fast iterative shrinkage-thresholding algorithm for linear inverse problems, SIAM journal on imaging sciences, 2 (2009), pp. 183-202.

[7] Z. Belhachmi, T. Glatz, And O. Scherzer, A direct method for photoacoustic tomography with inhomogeneous sound speed, Inverse Problems, 32 (2016), p. 045005.

[8] H. Brezis, Functional analysis, Sobolev spaces and partial differential equations, Springer, 2010 .

[9] P. Burgholzer, C. Hofer, G. Paltauf, G. Matt, M. Haltmeier, and O. SCHERZER, Thermoacoustic tomography using a fiber based Fabry-Perot interferometer as an integrating line detector, in Proc. of SPIE Vol, vol. 6086, 2006, pp. 60861N-1.

[10] P. Burgholzer, G. J. Matt, M. Haltmeier, and G. Paltauf, Exact and approximate imaging methods for photoacoustic tomography using an arbitrary detection surface, Physical Review E, 75 (2007), p. 046706.

[11] C. Clason and M. V. Klibanov, The quasi-reversibility method for thermoacoustic tomography in a heterogeneous medium, SIAM Journal on Scientific Computing, 30 (2008), pp. 1-23, 
[12] B. Cox, S. Kara, S. Arridge, And P. BeARd, k-space propagation models for acoustically heterogeneous media: Application to biomedical photoacoustics, J. Acoust. Soc. Am., 121 (2007), pp. 3453-3464.

[13] J. W. DANIEL, The conjugate gradient method for linear and nonlinear operator equations, SIAM Journal on Numerical Analysis, 4 (1967), pp. 10-26.

[14] X. L. Dean-Ben, A. Buehler, V. Ntziachristos, and D. Razansky, Accurate modelbased reconstruction algorithm for three-dimensional optoacoustic tomography, IEEE Transactions on Medical Imaging, 31 (2012), pp. 1922-1928.

[15] H. W. Engl, M. Hanke, And A. Neubauer, Regularization of inverse problems, vol. 375, Springer, 1996.

[16] O. G. ERnst, Minimal and orthogonal residual methods and their generalizations for solving linear operator equations, 2000. Habilitation thesis, TU Bergakademie Freiberg.

[17] L. C. Evans, Partial differential equations, vol. 19 of Graduate Studies in Mathematics, American Mathematical Society, Providence, RI, 1998.

[18] D. Finch, M. Haltmeier, And Rakesh, Inversion of spherical means and the wave equation in even dimensions, SIAM Journal on Applied Mathematics, 68 (2007), pp. 392412.

[19] D. Finch, S. K. PATCh, AND RAKeSh, Determining a function from its mean values over a family of spheres, SIAM Journal on Mathematical Analysis, 35 (2004), pp. 1213-1240 (electronic).

[20] Z. Fortuna, Some convergence properties of the conjugate gradient method in Hilbert space, SIAM Journal on Numerical Analysis, 16 (1979), pp. 380-384.

[21] J. FRIKel And E. T. Quinto, Artifacts in incomplete data tomography with applications to photoacoustic tomography and sonar, SIAM Journal on Applied Mathematics, 75 (2015), pp. $703-725$.

[22] R. Glowinski And S. LAPIn, Iterative solution of linear variational problems in Hilbert spaces: some conjugate gradients success stories, in Conjugate Gradient Algorithms and Finite Element Methods, Springer, 2004, pp. 223-245.

[23] M. Haltmeier, Inversion of circular means and the wave equation on convex planar domains, Computers \& Mathematics with Applications. An International Journal, 65 (2013), pp. 1025-1036.

[24] M. HaltmeieR, Universal inversion formulas for recovering a function from spherical means, SIAM Journal on Mathematical Analysis, 46 (2014), pp. 214-232.

[25] M. Haltmeier, O. Scherzer, P. Burgholzer, and G. Paltauf, Thermoacoustic computed tomography with large planar receivers, Inverse Problems, 20 (2004), p. 1663.

[26] M. Hanke, Conjugate gradient type methods for ill-posed problems, vol. 327, CRC Press, 1995. 
[27] R. M. HAYES, Iterative methods of solving linear problems on Hilbert space, Bulletin of the American Mathematical Society, 58 (1952), pp. 653-653.

[28] R. Herzog And E. SAChs, Superlinear convergence of Krylov subspace methods for selfadjoint problems in hilbert space, SIAM Journal on Numerical Analysis, 53 (2015), pp. 13041324 .

[29] E. Hestenes, M. R.And Stiefel, Methods of conjugate gradients for solving linear systems, Journal of Research of the National Bureau of Standards, 49 (1952).

[30] L. HöRmander, The analysis of linear partial differential operators. I, Classics in Mathematics, Springer-Verlage, Berlin, 2003.

[31] Y. HRIstova, Time reversal in thermoacoustic tomography-an error estimate, Inverse Problems, 25 (2009), pp. 055008, 14.

[32] Y. Hristova, P. Kuchment, and L. Nguyen, Reconstruction and time reversal in thermoacoustic tomography in acoustically homogeneous and inhomogeneous media, Inverse Problems, 24 (2008), pp. 055006, 25.

[33] C. Huang, K. Wang, L. Nie, and M. A. Wang, L. V.and Anastasio, Full-wave iterative image reconstruction in photoacoustic tomography with acoustically inhomogeneous media, IEEE Transactions on Medical Imaging, 32 (2013), pp. 1097-1110.

[34] W. J. KAmmerer AND M. Z. NASheD, On the convergence of the conjugate gradient method for singular linear operator equations, SIAM Journal on Numerical Analysis, 9 (1972), pp. 165-181.

[35] P. Kuchment, The Radon transform and medical imaging, vol. 85, SIAM, 2014.

[36] P. Kuchment and L. Kunyansky, Mathematics of thermoacoustic tomography, European Journal of Applied Mathematics, 19 (2008), pp. 191-224.

[37] L. A. Kunyansky, Explicit inversion formulae for the spherical mean Radon transform, Inverse Problems, 23 (2007), pp. 373-383.

[38] L. A. Kunyansky, A series solution and a fast algorithm for the inversion of the spherical mean radon transform, Inverse Problems, 23 (2007), p. S11.

[39] I. Lasiecka, J.-L. Lions, And R. TRiggiani, Nonhomogeneous boundary value problems for second order hyperbolic operators, J. Math. Pures Appl, 65 (1986), pp. 149-192.

[40] J. L. Lions And E. MAGEnes, Non-homogeneous boundary value problems and applications, vol. 1, Springer, 2012.

[41] A. K. Louis AND E. T. Quinto, Local tomographic methods in sonar, in Surveys on solution methods for inverse problems, Springer, Vienna, 2000, pp. 147-154.

[42] K.-A. Mardal and R. Winther, Preconditioning discretizations of systems of partial differential equations, Numerical Linear Algebra with Applications, 18 (2011), pp. 1-40. 
[43] T. D. Mast, L. P. Souriau, D. D. Liu, M. Tabei, A. I. Nachman, and R. C. Waag, A k-space method for large-scale models of wave propagation in tissue, IEEE Transactions on Ultrasonics, Ferroelectrics, and Frequency Control, 48 (2001), pp. 341-354.

[44] D. Modgil, M. Anastasio, and P. La RiviÈre, Image reconstruction in photoacoustic tomography with variable speed of sound using a higher-order geometrical acoustics approximation, Journal of Biomedical Optics, 15 (2010), p. 021308.

[45] F. NatTerer, Photo-acoustic inversion in convex domains, Inverse Problems Imaging, (2012).

[46] Y. Nesterov, Introductory lectures on convex optimization. applied optimization, vol. 87, 2004 .

[47] Y. Nesterov, A method for solving the convex programming problem with convergence rate $o\left(1 / k^{2}\right)$, Doklady Akademii Nauk, 269 (1983), pp. 543-547. in Russian.

[48] O. Nevanlinna, Convergence of iterations for linear equations, Birkhäuser, 2012.

[49] L. V. NGuYen, A family of inversion formulas in thermoacoustic tomography, Inverse Probl. Imaging, 3 (2009), pp. 649-675,

[50] L. V. NGUYen, On singularities and instability of reconstruction in thermoacoustic tomography, Tomography and inverse transport theory, Contemporary Mathematics, 559 (2011), pp. 163-170.

[51] L. V. NGuYen, On artifacts in limited data spherical Radon transform: Flat observation surfaces, SIAM J. Math. Analysis, 47 (2015), pp. 2984-3004,

[52] L. V. Nguyen and L. A. Kunyansky, A dissipative time reversal technique for photoacoustic tomography in a cavity, SIAM Journal on Imaging Sciences, 9 (2016), pp. 748-769.

[53] V. P. Palamodov, A uniform reconstruction formula in integral geometry, Inverse Problems, 28 (2012), p. 065014.

[54] G. Paltauf, P. Burgholzer, M. Haltmeier, and O. Scherzer, Thermoacoustic tomography using optical line detection, in European Conference on Biomedical Optics, Optical Society of America, 2005.

[55] J. Qian, P. Stefanov, G. Uhlmann, and H. Zhao, An efficient Neumann seriesbased algorithm for thermoacoustic and photoacoustic tomography with variable sound speed, SIAM Journal on Imaging Sciences, 4 (2011), pp. 850-883.

[56] A. Rosenthal, V. Ntziachristos, and D. Razansky, Acoustic inversion in optoacoustic tomography: A review, Current medical imaging reviews, 9 (2013), p. 318.

[57] P. Stefanov and G. Uhlmann, Thermoacoustic tomography with variable sound speed, Inverse Problems, 25 (2009), pp. 075011, 16.

[58] P. Stefanov and G. Uhlmann, Thermoacoustic tomography arising in brain imaging, Inverse Problems, 27 (2011), p. 045004. 
[59] P. Stefanov And Y. YAng, Multiwave tomography with reflectors: Landweber's iteration, ArXiv e-prints, (2016),

[60] M. TABei, T. D. Mast, And R. C. WAaG, A k-space method for coupled first-order acoustic propagation equations, Journal of the Acoustical Society of America, 111 (2002), pp. 53-63.

[61] M. TAYLOR, Pseudodifferential operators, volume 34 of Princeton Mathematical Series. 1981.

[62] B. E. Treeby And B. T. Cox, k-wave: Matlab toolbox for the simulation and reconstruction of photoacoustic wave fields, Journal of biomedical optics, 15 (2010), pp. 021314021314 .

[63] F. TRÈves, Introduction to pseudodifferential and Fourier integral operators. Vol. 2, Plenum Press, New York, 1980. Fourier integral operators.

[64] K. Wang And M. A. Anastasio, Photoacoustic and thermoacoustic tomography: image formation principles, in Handbook of Mathematical Methods in Imaging, Springer, 2011, pp. $781-815$.

[65] K. Wang, S. A. Ermilov, R. Su, H.-P. Brecht, A. A. Oraevsky, and M. A. AnASTASIO, An imaging model incorporating ultrasonic transducer properties for threedimensional optoacoustic tomography, IEEE transactions on medical imaging, 30 (2011), pp. 203-214.

[66] K. Wang, R. Su, A. A. Oraevsky, and M. A. Anastasio, Investigation of iterative image reconstruction in three-dimensional optoacoustic tomography, Physics in medicine and biology, 57 (2012), p. 5399.

[67] M. Xu, L. V. Wang, G. Ambartsoumian, and P. Kuchment, Limited view thermoacoustic tomography, in Photoacoustic imaging and spectroscopy, CRC Press, 2009, pp. pp. $61-73$.

[68] G. Zangerl, O. Scherzer, and M. Haltmeier, Circular integrating detectors in photo and thermoacoustic tomography, Inverse Problem Science and Engineering, 17 (2009), pp. 133-142. 